\title{
Kontsevich's conjecture on an algebraic formula for vanishing cycles of local systems
}

\author{
Claude Sabbah and Morihiko Saito
}

\begin{abstract}
For a local system and a function on a smooth complex algebraic variety, we give a proof of a conjecture of M. Kontsevich on a formula for the vanishing cycles using the twisted de Rham complex of the formal microlocalization of the corresponding locally free sheaf with integrable connection having regular singularity at infinity. We also prove its local version, which may be viewed as a natural generalization of a result of E. Brieskorn in the isolated singularity case. We then generalize these to the case of the de Rham complexes of regular holonomic D-modules where we have to use the tensor product with a certain sheaf of formal microlocal differential operators instead of the formal completion.
\end{abstract}

\section{Introduction}

Let $X$ be a smooth complex algebraic variety and let $f \in \Gamma\left(X, \mathcal{O}_{X}\right)$. Let $L$ be a $\mathbf{C}$-local system on $X$. For each $c \in \mathbf{C}$, we have the vanishing cycle sheaf complex $\varphi_{f-c} L$ on $X_{c}:=f^{-1}(c)$ with the monodromy $T_{c}$, as is defined by Deligne [Del73] (see also Notation below). Let $S=\mathbf{C}$ as a complex algebraic variety endowed with the natural coordinate $t$. Then $f$ is identified with a morphism $f: X \rightarrow S$ and $f^{*} t$ with the function $f$. Set $\partial_{t}:=\partial / \partial t$ and define

$$
\widehat{R}:=\mathbf{C} \llbracket \partial_{t}^{-1} \rrbracket, \quad \widehat{K}:=\mathbf{C}\left(\left(\partial_{t}^{-1}\right)\right)=\widehat{R}\left[\partial_{t}\right] .
$$

Let $\widehat{K}\langle t\rangle=\bigoplus_{i \in \mathbf{N}} \widehat{K} t^{i}$. This has a structure of a noncommutative ring by $\partial_{t} t-t \partial_{t}=1$. For a finite-dimensional $\mathbf{C}$-vector space $V$ with an automorphism $T$ and for $c \in \mathbf{C}$, define a $\widehat{K}\langle t\rangle$ module by

$$
\widehat{\mathcal{E}}(V, T)_{c}:=V\left(\left(\partial_{t}^{-1}\right)\right)=V \llbracket \partial_{t}^{-1} \rrbracket\left[\partial_{t}\right]
$$

with action of $t$ given by

$$
t\left(v \partial_{t}^{j}\right)=\left(-\frac{\log T}{2 \pi i}-j\right) v \partial_{t}^{j-1}+c v \partial_{t}^{j} \quad \text { for } v \in V, j \in \mathbf{Z},
$$

where the real part of the eigenvalues of $(2 \pi i)^{-1} \log T$ are contained in $[0,1)$.

Received 15 June 2013, accepted in final form 19 August 2013.

2010 Mathematics Subject Classification 14F10

Keywords: Twisted de Rham complex, regular holonomic D-module, formal microdifferential operator, vanishing cycles

This journal is (C) Foundation Compositio Mathematica 2014. This article is distributed with Open Access under the terms of the Creative Commons Attribution Non-Commercial License, which permits non-commercial reuse, distribution, and reproduction in any medium, provided that the original work is properly cited. For commercial re-use, please contact the Foundation Compositio Mathematica.

The first named author is supported by the grant ANR-08-BLAN-0317-01 of the Agence nationale de la recherche. The second named author is partially supported by Kakenhi 21540037. 


\section{SABbah And M. SAito}

Corresponding to the local system $L$, we have a locally free $\mathcal{O}_{X}$-module $\mathcal{M}$ with an integrable connection $\nabla$ having regular singularities at infinity [Del70]. Set $u:=\partial_{t}^{-1}$. We have a twisted de Rham complex

$$
\mathcal{M}((u))^{f} \otimes_{\mathcal{O}_{X}} \Omega_{X}^{\bullet}:=\operatorname{DR}_{X}\left(\mathcal{M}((u))^{f}\right)[-\operatorname{dim} X],
$$

where $\mathcal{M}((u))^{f}:=\mathcal{M} \llbracket u \rrbracket\left[u^{-1}\right]$ and the differential of $\operatorname{DR}_{X}\left(\mathcal{M}((u))^{f}\right)$ is given by

$$
\nabla-u^{-1} \otimes d f \text {. }
$$

Note that $\mathcal{M}((u))^{f}$ has a natural action of $\widehat{K}=\mathbf{C}((u))$ together with an action of $t$ defined by

$$
t\left(m \partial_{t}^{j}\right)=f m \partial_{t}^{j}-j m \partial_{t}^{j-1} \text { for } m \in \mathcal{M}, j \in \mathbf{Z} .
$$

In this paper we give a proof of the following result, which was conjectured by M. Kontsevich as a possible answer to a question raised in [KS11] concerning a definition of vanishing cycles for functions on smooth formal schemes. We also simplify some arguments of a different approach in $[$ Sab10].

Theorem 1. For $k \in \mathbf{Z}$, there are canonical isomorphisms as $\widehat{K}\langle t\rangle$-modules

$$
H^{k}\left(X, \mathcal{M}((u))^{f} \otimes_{\mathcal{O}_{X}} \Omega_{X}^{\bullet}\right)=\bigoplus_{c \in \mathbf{C}} \widehat{\mathcal{E}}\left(H^{k-1}\left(X_{c}, \varphi_{f-c} L\right), T_{c}^{(k-1)}\right)_{c},
$$

where $T_{c}^{(k-1)}$ is induced by $T_{c}$.

Note that the right-hand side is a finite direct sum since $\varphi_{f-c} L=0$ except for a finite number of $c$. Viewed as a formal meromorphic connection for the variable $u$, the above decomposition is a special case of the classical Levelt-Turrittin theorem. Let $\mathcal{V}^{k}$ denote the left-hand side of the formula. Theorem 1 means that, in order to calculate $\left(H^{k-1}\left(X_{c}, \varphi_{f-c} L\right), T_{c}^{(k-1)}\right)$, it is enough to find a finite dimensional $\mathbf{C}$-vector subspace $V^{k}$ of $\mathcal{V}^{k}$ such that

$$
\widehat{K} \otimes_{\mathbf{C}} V^{k} \stackrel{\sim}{\longrightarrow} \mathcal{V}^{k}, \quad t V^{k} \subset \widehat{R} V^{k},
$$

and moreover $A_{0}$ is semisimple and any two eigenvalues of $A_{1}(c, c)$ do not differ by an integer for each $c$. Here $\sum_{i \geqslant 0} A_{i} \partial_{t}^{-i}$ is the expansion of the action of $t$ with $A_{i} \in \operatorname{End}_{\mathbf{C}}\left(V^{k}\right), V^{k}=\bigoplus_{c} V_{c}^{k}$ is the eigenspace decomposition by the action of $A_{0}$, and $A_{1}(c, c)$ is the $\left(V_{c}^{k}, V_{c}^{k}\right)$-component of $A_{1}$. In this case, we have the following isomorphism (see Remark 3.6 below):

$$
\left(H^{k-1}\left(X_{c}, \varphi_{f-c} L\right), T_{c}^{(k-1)}\right)=\left(V_{c}^{k}, \exp \left(-2 \pi i A_{1}(c, c)\right)\right) .
$$

In case $f$ has only isolated singular points, this calculation is essentially equivalent to the one given by E. Brieskorn [Bri70] (see $\S 3.5$ below). We have a Čech calculation in the general case (see $\S 3.4$ below). In special cases, we have the following.

Corollary 1. If $X$ is affine, then the formula in Theorem 1 holds with the left-hand side replaced by the $k$-th cohomology of the complex whose $q$-th component is

$$
\Gamma\left(X, \mathcal{M} \otimes \mathcal{O}_{X} \Omega_{X}^{q}\right)((u))^{f},
$$

where the differential is induced by $\nabla-u^{-1} d f \wedge$.

Corollary 2. If $L=\mathbf{C}_{X}$ and $X$ is an affine open subvariety of $\mathbf{C}^{n}$, let $A_{X}$ be the affine ring of $X$ with $x_{1}, \ldots, x_{n}$ the coordinates of $\mathbf{C}^{n}$. Set $\partial_{x_{i}}:=\partial / \partial x_{i}$ and $f_{i}:=\partial f / \partial x_{i}$. The formula in Theorem 1 holds with the left-hand side replaced by

$$
H^{k} K^{\bullet}\left(A_{X}((u)) ; \partial_{x_{i}}-u^{-1} f_{i}(i \in[1, n]),\right.
$$

which is the cohomology of the Koszul complex for the action of $\partial_{x_{i}}-u^{-1} f_{i}(i \in[1, n])$ on $A_{X}((u))$. 


\section{KONTSEVICH'S CONJECTURE}

What is interesting in Theorem 1 and its corollaries is that we have only the contributions of the vanishing cycles of $f$ inside $X$ and no contributions of the vanishing cycles at infinity on the right-hand side of the equality. This might be rather surprising, since the cohomological direct images $\mathcal{H}^{j} f_{*} \mathcal{M}$ of $\mathcal{M}$ as $\mathcal{D}$-modules are defined by

$$
\mathcal{H}^{j} f_{*} \mathcal{M}:=\mathcal{H}^{j} f_{\bullet}\left(\mathcal{M}\left[\partial_{t}\right]^{f} \otimes_{\mathcal{O}_{X}} \Omega_{X}^{\bullet}[\operatorname{dim} X]\right),
$$

where $f_{\bullet}$ denotes the sheaf-theoretic direct image and we have $\partial_{t}=u^{-1}$, so that $\mathcal{M}((u))^{f}$ is isomorphic to the formal microlocalization of $\mathcal{M}\left[\partial_{t}\right]^{f}$. Note that the vanishing cycles of the cohomological direct images $\mathcal{H}^{j} f_{*} \mathcal{M}$ have the contributions from the vanishing cycles at infinity in general. So these imply that one cannot apply the formal microlocalization after taking the direct image of $\mathcal{M}$ by $f$ as a $\mathcal{D}$-module. Also note that the vanishing cycle functor does not necessarily commute with the direct image under a nonproper morphism $f$ because of the problem of singularities at infinity of $f$.

Let $j: X \hookrightarrow \bar{X}$ be a smooth compactification such that $f$ induces a morphism $\bar{f}: \bar{X} \rightarrow \mathbf{P}^{1}$. Set $\overline{\mathcal{M}}=j_{*} \mathcal{M}$. Using GAGA we can show the following (see also [Sab10]).

Proposition 1. For $k \in \mathbf{Z}$, there are canonical isomorphisms of $\widehat{K}\langle t\rangle$-modules

$$
H^{k}\left(\bar{X}, \overline{\mathcal{M}}((u))^{f} \otimes_{\mathcal{O}_{\bar{X}}} \Omega_{\bar{X}}^{\bullet} \stackrel{\sim}{\longrightarrow} H^{k}\left(\bar{X}^{\text {an }}, \overline{\mathcal{M}}^{\text {an }}((u))^{f} \otimes_{\mathcal{O}_{\bar{X}} \text { an }} \Omega_{\bar{X}^{\text {an }}}\right) .\right.
$$

Note that the noncommutativity of cohomology and inductive limit does not cause a problem for the construction of the above canonical morphism by using the continuous morphism $\rho: \bar{X}^{\text {an }} \rightarrow \bar{X}$, see $\S 3.1$. By Proposition 1 , the proof of Theorem 1 can be reduced to local analytic calculations on $\bar{X}^{\text {an }}$. So we will consider only the underlying complex manifolds, and the upperscript ${ }^{\text {an }}$ will be omitted from now on in this paper (except for $\S \S 2.1,2.3$ and 3.1). Moreover, it is enough to work mainly on $X$ by Proposition 4 below.

We also have the twisted de Rham complex $\mathcal{M}\left[\partial_{t}, \partial_{t}^{-1}\right]^{f} \otimes_{\mathcal{O}_{X}} \Omega_{X}^{\bullet}$ associated with the algebraic localization $\mathcal{M}\left[\partial_{t}, \partial_{t}^{-1}\right]^{f}$. Consider its cohomology sheaves

$$
\mathcal{H}^{j}\left(\mathcal{M}\left[\partial_{t}, \partial_{t}^{-1}\right]^{f} \otimes_{\mathcal{O}_{X}} \Omega_{X}^{\bullet}\right)
$$

These are constructible sheaves of Gauss-Manin systems in one variable (up to the division by $\mathbf{C}[t]$ for $j=1$ ), see [BS07]. We show that the topology on their stalks at each $x \in X$ induced by the $u$-adic topology on $\mathcal{M}[u]^{f}$ coincides with the one induced by the $\partial_{t}^{-1}$-adic topology on the Brieskorn lattices of the Gauss-Manin systems by using the finiteness theorem on the order of the torsion of Brieskorn modules proved in loc. cit. We then get the following.

Proposition 2. There is a canonical quasi-isomorphism of complexes of $f^{-1} \widehat{\mathcal{E}}_{S^{-m o d u l e s}}$

$$
f^{-1} \widehat{\mathcal{E}}_{S} \otimes_{f^{-1} \mathcal{D}_{S}}\left(\mathcal{M}\left[\partial_{t}\right]^{f} \otimes_{\mathcal{O}_{X}} \Omega_{X}^{\bullet}\right) \stackrel{\sim}{\longrightarrow} \mathcal{M}\left(\left(\partial_{t}^{-1}\right)\right)^{f} \otimes_{\mathcal{O}_{X}} \Omega_{X}^{\bullet},
$$

where $\mathcal{M}\left[\partial_{t}\right]^{f}$ is the direct image of $\mathcal{M}$ as an analytic $\mathcal{D}$-module by the graph embedding of $f$ and $\widehat{\mathcal{E}}_{S}$ is the ring of formal microdifferential operators on the complex manifold $S=\mathbf{C}$, that is, $\widehat{\mathcal{E}}_{S}=\mathcal{O}_{S}\left(\left(\partial_{t}^{-1}\right)\right)$ forgetting the multiplicative structure.

Here we also use the assertion that the filtration $V$ of Kashiwara and Malgrange on $\mathcal{M}\left[\partial_{t}\right]^{f}$ induces the $V$-filtration on each stalk of the constructible cohomology sheaves of the Gauss-Manin systems by taking the relative de Rham complex, see (1.3.3) below.

Using Proposition 2 we get the following local analytic version of Theorem 1 for local systems $L$ and holomorphic functions $f$ on complex manifolds $X$, where $\mathcal{M}=\mathcal{O}_{X} \otimes_{\mathbf{C}} L$ in the analytic case. 


\section{SABbah And M. SAito}

THEOREM 2. For $k \in \mathbf{Z}$, there are canonical isomorphisms of analytic constructible sheaves of $\widehat{K}\langle t\rangle$-modules on $X_{c}$

$$
\left.\mathcal{H}^{k+1}\left(\mathcal{M}((u))^{f} \otimes_{\mathcal{O}_{X}} \Omega_{X}^{\bullet}\right)\right|_{X_{c}}=\widehat{\mathcal{E}}\left(\mathcal{H}^{k} \varphi_{f-c} L, T_{c}^{(k-1)}\right)_{c},
$$

where $\widehat{\mathcal{E}}(*)_{c}$ on the right-hand side is naturally extended to the case of constructible sheaves.

This would not be very surprising to the specialists in view of [BS07] and also [Sai88a] (see Remark 1.7(i) and Remark 3.6 below). If $f$ has only isolated singular points, then it is essentially equivalent to the calculation of Brieskorn [Bri70] explained after Theorem 1.

For the proof of Theorem 1, we have a passage from local to global, and need the following.

Proposition 3. The filtration $V$ of Kashiwara and Malgrange on $\mathcal{M}\left[\partial_{t}\right]^{f}$ along $t=c$ induces the filtration $V$ on the global analytic cohomology $H^{k}\left(X_{c},\left.\mathcal{M}\left[\partial_{t}\right]^{f} \otimes_{\mathcal{O}_{X}} \Omega_{X}^{\bullet}\right|_{X_{c}}\right)$ in a strict way, that is, $\mathrm{Gr}_{V}^{\alpha}$ commutes with the global cohomology.

This is proved by using a filtered spectral sequence which is a priori defined in an abelian category containing the exact category of filtered vector spaces, see $\S 3.2$ below.

For the proof of Theorem 1, there still remains the following key proposition.

Proposition 4. We have a canonical analytic quasi-isomorphism

$$
\overline{\mathcal{M}}((u))^{f} \otimes \mathcal{O}_{\bar{X}} \Omega_{\bar{X}}^{\bullet} \stackrel{\sim}{\longrightarrow} \mathbf{R} j_{*}\left(\mathcal{M}((u))^{f} \otimes \mathcal{O}_{X} \Omega_{X}^{\bullet}\right) .
$$

Note that the right-hand side is supported on a union of a finite number of $\bar{X}_{c}(c \in \mathbf{C})$ by Theorem 2, and that this implies the vanishing of the restriction of the left-hand side to $\bar{X}_{\infty}$. The proof of Proposition 4 is reduced to the case where the union of $D:=\bar{X} \backslash X$ and the singular $X_{c}$ is a divisor with normal crossings on $\bar{X}$. Indeed, for a proper morphism of complex manifolds $\pi: X^{\prime} \rightarrow X$, the canonical morphism $L \rightarrow \mathbf{R} \pi_{*} \pi^{*} L$ splits by applying this morphism to the dual of $L$ and using Verdier duality. Here we use the direct image of differential complexes rather than that of $\mathcal{D}$-modules to simplify the argument. For the proof of Proposition 4 in the normal crossing case, we prove a sufficient condition for the commutativity of the global section functor and the inductive limit in $\S 1.8$, and apply this to the filtration $G$ used in the proof of Theorem 2.

In this paper we also show the following.

TheOREM 3. Theorems 1 and 2 remain valid by replacing $\mathcal{M}, L$, and $\mathcal{M}((u))^{f}$ with, respectively, a bounded complex of algebraic regular holonomic $\mathcal{D}_{X}$-modules $\mathcal{M}^{\bullet}, \mathrm{DR}_{X}\left(\mathcal{M}^{\bullet}\right)[-\operatorname{dim} X]$, and $\mathcal{M}^{\bullet} \otimes_{\mathcal{O}_{X}} \mathcal{O}_{X}((u))^{f}$.

Here the replacement of $\mathcal{M}((u))^{f}$ by $\mathcal{M}^{\bullet} \otimes_{\mathcal{O}_{X}} \mathcal{O}_{X}((u))^{f}$ is quite essential. Indeed, we cannot get a correct result if we apply the same definition as in the local system case. This is a quite subtle point of the theory, and is related to the noncommutativity of inductive limit and projective limit. Using canonical isomorphisms, the proof of Theorem 3 can be reduced to the case where $\mathcal{M}$ is a locally free $\mathcal{O}_{X}(* E)$-module having a regular singular connection. Here $E$ is a divisor on $X$, called the interior divisor. Also note that, setting $\overline{\mathcal{M}}=j_{*} \mathcal{M}$ with $j: X \hookrightarrow \bar{X}$ as above, the construction of $\overline{\mathcal{M}}((u))^{f}$ along the interior divisor $E$ is quite different from the one along the exterior divisor $D:=\bar{X} \backslash X$, see Example 2.2. We prove Theorem 3 after showing Theorems 1 and 2 in the local system case since many readers would be interested mainly in the original conjecture of Kontsevich in this special case. 


\section{KontSEVICH's CONJECTURE}

We thank the referee for useful comments to improve the paper.

In Section 1 we review some basics about the sheaves of Gauss-Manin systems and Brieskorn modules, and then prove Theorem 2 after showing Proposition 2. In Section 2 we recall some basics of formal microlocalization, and prove Proposition 4 by reducing to the normal crossing case. In Section 3 we prove Theorem 1 after showing Propositions 1 and 3. In Section 4 we show how to generalize the arguments in the previous sections in order to prove Theorem 3.

\section{Notation}

The nearby and vanishing cycle functors in [Del73] are denoted respectively by $\psi_{f}$ and $\varphi_{f}$. If $\psi_{f} \mathcal{F}$ is a (shifted) perverse sheaf and $\mathcal{F}$ has $\mathbf{C}$-coefficients, $\psi_{f, \lambda} \mathcal{F}$ denotes the $\lambda$-eigen-subsheaf of $\psi_{f} \mathcal{F}$ for the semisimple part $T_{s}$ of the monodromy $T$, that is, $\psi_{f, \lambda} \mathcal{F}:=\operatorname{Ker}\left(T_{s}-\lambda\right) \subset \psi_{f} \mathcal{F}$, and similarly for $\varphi_{f, \lambda} \mathcal{F}$.

\section{Sheaves of Gauss-Manin systems and Brieskorn modules}

In this section we review some basics about the sheaves of Gauss-Manin systems and Brieskorn modules, and then prove Theorem 2 after showing Proposition 2. Here we treat only complex manifolds and analytic sheaves.

\subsection{Regular holonomic $\mathcal{D}_{S, 0}$-modules}

Let $S=\mathbf{C}$ as a complex manifold. Let $t$ be the coordinate. Set $\partial_{t}=\partial / \partial t$. For a regular holonomic left $\mathcal{D}_{S, 0}$-module $M$ and $\alpha \in \mathbf{C}$, define

$$
M^{\alpha}:=\operatorname{Ker}\left(\left(t \partial_{t}-\alpha\right)^{k}: M \rightarrow M\right) \quad(k \gg 0) .
$$

For any $\alpha \in \mathbf{C}$, the actions of $t$ and $\partial_{t}$ on $M$ induce the morphisms

$$
t: M^{\alpha} \rightarrow M^{\alpha+1}, \quad \partial_{t}: M^{\alpha+1} \rightarrow M^{\alpha},
$$

which are bijections for $\alpha \neq-1$. We have the canonical inclusions

$$
\bigoplus_{\alpha \in \mathbf{C}} M^{\alpha} \subset M \subset \prod_{\alpha \in \mathbf{C}} M^{\alpha} .
$$

The last inclusion follows from the fact that $\bigoplus_{\alpha \in \mathbf{C}} M^{\alpha}$ generates $M$ over $\mathcal{O}_{S, 0}=\mathbf{C}\{t\}$. The latter can be proved by using the classical theory of differential equations of one variable with regular singularities, see for example [Del70]. (Note that (1.1.2) does not hold in the higher dimensional case unless $M$ is isomorphic to its Verdier specialization along the hypersurface.)

Take an extension of $M$ to a coherent $\mathcal{D}_{S}$-module defined on a sufficiently small open neighborhood of $0 \in S$, which is also denoted by $M$. We then get a perverse sheaf $\operatorname{DR}_{S}(M)$, replacing $S$ with a neighborhood of 0. By [Kas83], [Mal83], there are canonical isomorphisms of $\mathbf{C}$-vector spaces for $\lambda=\exp (-2 \pi \alpha)$ :

$$
M^{\alpha}= \begin{cases}\psi_{t, \lambda} \operatorname{DR}_{S}(M)[-1] & \text { if } \alpha \notin \mathbf{Z}_{\leqslant-1}, \\ \varphi_{t, \lambda} \operatorname{DR}_{S}(M)[-1] & \text { if } \alpha \notin \mathbf{Z}_{\geqslant 0},\end{cases}
$$

where $\mathbf{Z}_{\leqslant k}:=\{i \in \mathbf{Z} \mid i \leqslant k\}$, and similarly for $\mathbf{Z}_{\geqslant k}$. (For $\psi_{t, \lambda}, \varphi_{t, \lambda}$, see Notation at the end of the introduction.) Set

$$
\Lambda:=\{\alpha \in \mathbf{C} \mid \operatorname{Re} \alpha \in[0,1)\}
$$




\section{Sabbah And M. Saito}

We have canonical isomorphisms of $\mathbf{C}$-vector spaces

$$
\psi_{t} \mathrm{DR}_{S}(M)[-1]=\bigoplus_{\alpha \in \Lambda} M^{\alpha}, \quad \varphi_{t} \mathrm{DR}_{S}(M)[-1]=\bigoplus_{\alpha \in \Lambda-1} M^{\alpha} .
$$

Indeed, let $V$ denote the $V$-filtration of Kashiwara and Malgrange indexed decreasingly by $\mathbf{Z}$ so that the eigenvalues of the action of $t \partial_{t}$ on $\operatorname{Gr}_{V}^{i} M$ are contained in $\Lambda+i$, see loc. cit. Then

$$
V^{i} M=M \cap \prod_{\operatorname{Re} \alpha \geqslant i} M^{\alpha} .
$$

This implies the canonical splittings (using the coordinate $t$ ) for $i>j$ :

$$
V^{j} M=\prod_{j \leqslant \operatorname{Re} \alpha<i} M^{\alpha} \oplus V^{i} M .
$$

\subsection{Microlocalizations}

Let $\mathcal{E}_{S}$ be the ring of microdifferential operators on $P^{*} S(=S)$ and let $\widehat{\mathcal{E}}_{S}$ be the ring of formal ones, see [SKK73], [Kas03]. Here the projective cotangent bundle $P^{*} S$ is identified with $S$ since $\operatorname{dim} S=1$. Forgetting the multiplicative structure, we have

$$
\widehat{\mathcal{E}}_{S}=\mathcal{O}_{S}\left(\left(\partial_{t}^{-1}\right)\right)\left(:=\mathcal{O}_{S} \llbracket \partial_{t}^{-1} \rrbracket\left[\partial_{t}\right]\right) .
$$

It is known that $\mathcal{E}_{S}$ and $\widehat{\mathcal{E}}_{S}$ are flat over $\mathcal{D}_{S}$, see loc. cit. So the tensor with $\mathcal{E}_{S, 0}$ or $\widehat{\mathcal{E}}_{S, 0}$ over $\mathcal{D}_{S, 0}$ is an exact functor. Set

$$
\begin{aligned}
& R:=\mathbf{C}\left\{\left\{\partial_{t}^{-1}\right\}\right\}\left(:=\left\{\sum_{i \in \mathbf{N}} a_{i} \partial_{t}^{-i}\left|\sum_{i \in \mathbf{N}}\right| a_{i} \mid r^{i} / i !<\infty \text { for some } r>0\right\}\right), \\
& K:=R\left[\partial_{t}\right], \quad \widehat{R}=\mathbf{C} \llbracket \partial_{t}^{-1} \rrbracket, \quad \widehat{K}:=\widehat{R}\left[\partial_{t}\right]=\mathbf{C}\left(\left(\partial_{t}^{-1}\right)\right) .
\end{aligned}
$$

For $M$ as in $\S 1.1$, we then get the canonical isomorphisms

$$
\begin{aligned}
& \mathcal{E}_{S, 0} \otimes_{\mathcal{D}_{S, 0}} M \stackrel{\sim}{\longrightarrow} \bigoplus_{\alpha \in \Lambda} K \otimes_{\mathbf{C}} M^{\alpha}, \\
& \widehat{\mathcal{E}}_{S, 0} \otimes_{\mathcal{D}_{S, 0}} M \stackrel{\sim}{\longrightarrow} \bigoplus_{\alpha \in \Lambda} \widehat{K} \otimes_{\mathbf{C}} M^{\alpha} .
\end{aligned}
$$

To define the action of $\mathcal{E}_{S, 0}, \widehat{\mathcal{E}}_{S, 0}$ on the right-hand side, we use the expression

$$
P=\sum_{j \in \mathbf{Z}} \sum_{i=0}^{m-j} a_{i, j}\left(t \partial_{t}\right)^{i} \partial_{t}^{j} \quad\left(a_{i, j} \in \mathbf{C}\right) \quad \text { for } P \in F_{m} \mathcal{E}_{S, 0} \text { or } F_{m} \widehat{\mathcal{E}}_{S, 0},
$$

where $F$ is the filtration by the order of $\partial_{t}$. This induces the canonical morphisms in (1.2.3) by using (1.1.2). These are isomorphisms by reducing to the case $M$ simple, that is, $M=\mathcal{D}_{S, 0} / \mathcal{D}_{S, 0} Q$ with $Q$ either $\partial_{t}$ or $t$ or $t \partial_{t}-\alpha(\alpha \in \Lambda \backslash\{0\})$.

\subsection{Sheaves of Gauss-Manin systems}

Let $f$ be a nonconstant holomorphic function on a complex manifold $X$. Let $i_{f}: X \rightarrow X \times S$ be the graph embedding by $f$. Let $\mathcal{B}_{f}$ be the direct image of $\mathcal{O}_{X}$ by $i_{f}$ as a left $\mathcal{D}_{X}$-module. Then

$$
\mathcal{B}_{f}=\mathcal{O}_{X}\left[\partial_{t}\right]^{f}
$$

where the sheaf-theoretic direct image by $i_{f}$ (that is, the zero extension) is omitted to simplify the notation, and ${ }^{f}$ means that the actions of $t$ and vector fields $\xi$ on $X$ are twisted so that

$$
\begin{aligned}
\xi\left(g \partial_{t}^{i}\right) & =\xi g \partial_{t}^{i}-(\xi f) g \partial_{t}^{i+1} \quad \text { for } g \in \mathcal{O}_{X}, \\
t\left(g \partial_{t}^{i}\right) & =f g \partial_{t}^{i}-i g \partial_{t}^{i-1} .
\end{aligned}
$$

(Here the delta function $\delta(f-t)$ is actually omitted after $\partial_{t}^{i}$.) Set $X_{0}=f^{-1}(0)$, and define

$$
\mathcal{K}_{f}^{\bullet}=\left.\mathrm{DR}_{X \times S / S}\left(\mathcal{B}_{f}\right)[-\operatorname{dim} X]\right|_{X_{0}} .
$$




\section{KONTSEVICH'S CONJECTURE}

This is a complex whose $i$-th term is $\left.\Omega_{X}^{i}\left[\partial_{t}\right]\right|_{X_{0}}$ and whose differential is given by

$$
d\left(\omega \partial_{t}^{i}\right)=(d \omega) \partial_{t}^{i}-(d f \wedge \omega) \partial_{t}^{i+1} \quad \text { for } \omega \in \Omega_{X}^{i} .
$$

Set

$$
\mathcal{G}_{f}^{i}:=\mathcal{H}^{i} \mathcal{K}_{f}^{\bullet},
$$

where $\mathcal{H}^{i}$ denotes the cohomology sheaf of a sheaf complex. We call $\mathcal{G}_{f}^{i}$ the sheaf of Gauss-Manin systems associated with $f$. It is a sheaf of regular holonomic left $\mathcal{D}_{S, 0}$-modules.

For $i=1$, we see that $\omega_{0}:=d f \in \mathcal{G}_{f}^{1}$ is annihilated by $\partial_{t}$ (that is, $d f \partial_{t}=0$ in $\mathcal{G}_{f}^{1}$ ), considering the image of 1 by $d$. So $\mathcal{D}_{S, 0} \omega_{0}=\mathbf{C}\{t\} \omega_{0}$. We can see that it is a free $\mathbf{C}\{t\}$-module of rank 1 by using $\left[\partial_{t}, t^{i}\right]=i t^{i-1}$ inductively. Define the sheaf of reduced Gauss-Manin systems $\widetilde{\mathcal{G}}_{f}^{i}$ by

$$
\widetilde{\mathcal{G}}_{f}^{i}:= \begin{cases}\mathcal{G}_{f}^{i} & \text { if } i \neq 1, \\ \mathcal{G}_{f}^{1} / \mathbf{C}\{t\} \omega_{0} & \text { if } i=1 .\end{cases}
$$

It is well known that the stalks $\mathcal{G}_{f, x}^{i}$ are regular holonomic $\mathcal{D}_{S, 0}$-modules, and in the notation of $\S 1$, we moreover have canonical isomorphisms of constructible sheaves for $\lambda=\exp (-2 \pi i \alpha)$ :

$$
\left(\mathcal{G}_{f}^{i+1}\right)^{\alpha}= \begin{cases}\mathcal{H}^{i} \psi_{f, \lambda} \mathbf{C}_{X} & \text { if } \alpha \notin \mathbf{Z}_{\leqslant 0}, \\ \mathcal{H}^{i} \varphi_{f, \lambda} \mathbf{C}_{X} & \text { if } \alpha \notin \mathbf{Z}_{\geqslant 1} .\end{cases}
$$

For $\psi_{f, \lambda} \mathbf{C}_{X}, \varphi_{f, \lambda} \mathbf{C}_{X}$, see Notation at the end of the introduction.

Let $V$ denote the $V$-filtration of Kashiwara [Kas83] and Malgrange [Mal83] on $\mathcal{B}_{f}$ such that the roots of the minimal polynomial of the action of $t \partial_{t}$ on $\operatorname{Gr}_{V}^{i} \mathcal{B}_{f}$ are contained in $\Lambda+i$, where $\Lambda$ is as in (1.1.4). Then (1.3.2) is reduced to the assertion that the $V$-filtration on $\mathcal{G}_{f, x}^{i}$ is strictly induced by the $V$-filtration on $\mathcal{B}_{f}$, that is,

$$
V^{\alpha} \mathcal{G}_{f, x}^{i}=\operatorname{Im}\left(\mathcal{H}^{i} V^{\alpha} \mathcal{K}_{f, x}^{\bullet} \hookrightarrow \mathcal{H}^{i} \mathcal{K}_{f, x}^{\bullet}\right),
$$

where "strictly" means the injectivity of the morphism from $\mathcal{H}^{i} V^{\alpha} \mathcal{K}_{f, x}^{\bullet}$. The proof of (1.3.3) is further reduced to the assertion that the induced filtration on $\mathcal{G}_{f, x}^{i}$ consists of finite $\mathcal{O}_{S, 0^{-}}$ modules since the other conditions of the $V$-filtration can be proved easily by using the action of $t \partial_{t}$ together with this property. Then the assertion follows from [Sai88b], Prop. 3.4 .8 and Lemma 3.4.9. For the proof of (1.3.2) we also use the inclusion of Milnor tubes for $x \in X_{0}$ and $x^{\prime} \in X_{0}$ sufficiently near $x$ to show the compatibility of the isomorphism (1.3.2) with the sheaf structure.

By definition there is a distinguished triangle

$$
\mathbf{C}_{X_{0}} \rightarrow \psi_{f} \mathbf{C}_{X} \rightarrow \varphi_{f} \mathbf{C}_{X} \rightarrow
$$

In particular, $\mathcal{H}^{i} \psi_{f, \lambda} \mathbf{C}_{X}=\mathcal{H}^{i} \varphi_{f, \lambda} \mathbf{C}_{X}$ if $i \neq 0$ or $\lambda \neq 1$.

By (1.3.2) and (1.3.4), $\widetilde{\mathcal{G}}_{f}^{i}$ is the microlocalization of $\mathcal{G}_{f}^{i}$, that is,

$$
\widetilde{\mathcal{G}}_{f}^{i}=\mathcal{E}_{S, 0} \otimes_{\mathcal{D}_{S, 0}} \mathcal{G}_{f}^{i} \quad \text { for any } i \in \mathbf{Z},
$$

and the $\widetilde{\mathcal{G}}_{f}^{i}$ are finite dimensional $K$-vector spaces, where $K$ is as in (1.2.2).

\subsection{Sheaves of Brieskorn modules}

With the notation of $\S 1.3$, let $\mathcal{A}_{f}^{\bullet}$ be the complex defined by

$$
\mathcal{A}_{f}^{i}:=\left.\operatorname{Ker}\left(d f \wedge: \Omega_{X}^{i} \rightarrow \Omega_{X}^{i+1}\right)\right|_{X_{0}},
$$




\section{Sabbah and M. Saito}

with differential induced by $d$. There is a natural inclusion

$$
\mathcal{A}_{f}^{\bullet} \rightarrow \mathcal{K}_{f}^{\bullet}
$$

Let

$$
\mathcal{H}_{f}^{i}=\mathcal{H}^{i} \mathcal{A}_{f}^{\bullet} .
$$

Note that we have to take the restriction to $X_{0}$ in order to define the action of $\partial_{t}^{-1}$ on $\mathcal{H}^{1} \mathcal{A}_{f}^{\bullet}$, see [BS07]. We will call $\mathcal{H}_{f}^{i}$ the sheaf of Brieskorn modules associated with $f$. The inclusion (1.4.1) induces the canonical morphism

$$
\mathcal{H}_{f}^{i} \rightarrow \mathcal{G}_{f}^{i}
$$

Since the differential is $f^{-1} \mathcal{O}_{S}$-linear, there is a natural structure of a $\mathbf{C}\{t\}$-module on $\mathcal{H}_{f}^{i}$. We have the action of $\partial_{t}^{-1}$ on $\mathcal{H}_{f}^{i}$ defined by

$$
\partial_{t}^{-1} \omega=d f \wedge \eta \quad \text { with } \quad d \eta=\omega .
$$

This action is well defined. For $i \neq 1$, the ambiguity of $\eta$ is given by $d \eta^{\prime}$, but we have $d f \wedge d \eta^{\prime}=$ $-d\left(d f \wedge \eta^{\prime}\right)$. For $i=1$, the ambiguity of $\eta \in \mathcal{O}_{X}$ is given by $\mathbf{C}$, and we have a canonical choice of $\eta$ assuming that the restriction of $\eta$ to $X_{0}$ vanishes (this is allowed because $d f \wedge d \eta=0$ ).

For $i=1$, we see that $\omega_{0}:=d f \otimes 1$ in $\S 1.3$ belongs to $\mathcal{H}_{f}^{1}$. Here we can easily verify that $\mathcal{H}_{f}^{1} \rightarrow \mathcal{G}_{f}^{1}$ is injective, see the proof of Th. 2.2 in [BS07]. We define the sheaf of reduced Brieskorn modules $\widetilde{\mathcal{H}}_{f}^{i}$ by

$$
\widetilde{\mathcal{H}}_{f}^{i}:= \begin{cases}\mathcal{H}_{f}^{i} & \text { if } i \neq 1, \\ \mathcal{H}_{f}^{1} / \mathbf{C}\{t\} \omega_{0} & \text { if } i=1 .\end{cases}
$$

Denote by $\mathcal{H}_{f, \text { tors }}^{i}$ the $\partial_{t}^{-1}$-torsion part of $\mathcal{H}_{f}^{i}$, and set $\mathcal{H}_{f \text {,free }}^{i}:=\mathcal{H}_{f}^{i} / \mathcal{H}_{f \text {,tors }}^{i}$ (similarly for $\left.\widetilde{\mathcal{H}}_{f, \text { tors }}^{i}, \widetilde{\mathcal{H}}_{f, \text { free }}^{i}\right)$. Let $R, K$ be as in (1.2.2). By [BS07], Th. 1, we have

$$
\begin{aligned}
& \mathcal{H}_{f, \text { tors }}^{i}=\operatorname{Ker}\left(\mathcal{H}_{f}^{i} \rightarrow \mathcal{G}_{f}^{i}\right), \\
& \left.\partial_{t}^{-p} \widetilde{\mathcal{H}}_{f, \text { tors }}^{i}=0 \text { if } p \gg 0 \text { (locally on } X_{0}\right), \\
& \widetilde{\mathcal{H}}_{f, \text { free }, x}^{i} \cong \bigoplus^{\mu_{i, x}} R \text { with } \mu_{i, x}:=\operatorname{dim}_{K} \widetilde{\mathcal{G}}_{f, x}^{i} .
\end{aligned}
$$

\subsection{Proof of Proposition 2}

We may assume that $c=0$ and moreover that

$$
\mathcal{M}=\mathcal{O}_{X}
$$

since the assertion is analytic and local on $X$. By (1.2.3) and (1.3.5) it is enough to show a canonical isomorphism of sheaves of $\widehat{\mathcal{E}}_{S, 0}$-modules

$$
\left.\mathcal{H}^{i}\left(\mathcal{O}_{X}\left(\left(\partial_{t}^{-1}\right)\right)^{f} \otimes_{\mathcal{O}_{X}} \Omega_{X}^{\bullet}\right)\right|_{X_{0}}=\widehat{K} \otimes_{K} \widetilde{\mathcal{G}}_{f}^{i},
$$

in a way compatible with the canonical morphisms from $\mathcal{G}_{f}^{i}$.

With the notation of $\S \S 1.3-1.4$, consider the algebraic microlocalization $\mathcal{K}_{f}^{\bullet}\left[\partial_{t}^{-1}\right]$ and define increasing filtrations $F_{\bullet}$ and $G$ • by setting

$$
\begin{aligned}
& F_{k} \mathcal{K}_{f}^{p}\left[\partial_{t}^{-1}\right]:=\left.\left(\bigoplus_{i \leqslant k+p} \Omega_{X}^{p} \partial_{t}^{i}\right)\right|_{X_{0}}, \\
& G_{k} \mathcal{K}_{f}^{p}\left[\partial_{t}^{-1}\right]:=\left.\mathcal{A}_{f}^{p} \partial_{t}^{k} \oplus\left(\bigoplus_{i<k} \Omega_{X}^{p} \partial_{t}^{i}\right)\right|_{X_{0}} .
\end{aligned}
$$




\section{KONTSEVICH'S CONJECTURE}

Note that $\mathcal{A}_{f}^{\bullet}$ is a subcomplex of $G_{0} \mathcal{K}_{f}^{\bullet}\left[\partial_{t}^{-1}\right]$. The formal microlocalization of $\mathcal{K}_{f}^{\bullet}$ can be defined by $F$ and also by $G$, that is,

$$
\left.\left(\mathcal{O}_{X}\left(\left(\partial_{t}^{-1}\right)\right)^{f} \otimes_{\mathcal{O}_{X}} \Omega_{X}^{\bullet}\right)\right|_{X_{0}}=\underset{p}{\lim }\left(\underset{q}{\lim }\left(F_{p} / F_{q}\right) \mathcal{K}_{f}^{\bullet}\left[\partial_{t}^{-1}\right]\right)
$$

where $F$ can be replaced with $G$ since these are cofinal with each other.

We have a canonical morphism between the short exact sequences

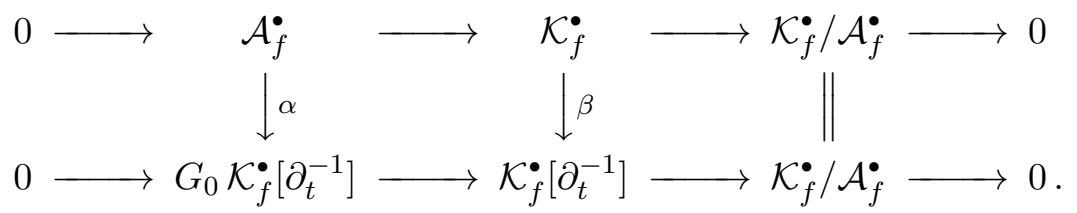

Using the morphism between the associated long exact sequences, for any $i$ we get the canonical isomorphisms

$$
\mathcal{H}^{i}\left(G_{0} \mathcal{K}_{f}^{\bullet}\left[\partial_{t}^{-1}\right]\right)=\widetilde{\mathcal{H}}_{f}^{i},
$$

where the $\widetilde{\mathcal{H}}_{f}^{i}$ are as in (1.4.4). Indeed, consider the long exact sequence associated with the first short exact sequence. Dividing $\mathcal{H}_{f}^{1}=\mathcal{H}^{1} \mathcal{A}_{f}^{\bullet}$ and $\mathcal{G}_{f}^{1}=\mathcal{H}^{1} \mathcal{K}_{f}^{\bullet}$ by $\mathbf{C}[t] \omega_{0}$, we still get a long exact sequence. This is isomorphic to the long exact sequence associated with the second short exact sequence by the five lemma. Indeed, $\mathbf{C}[t] \omega_{0} \subset \mathcal{G}_{f}^{1}$ is the subsheaf annihilated by the localization by $\partial_{t}$ by (1.3.5) and the action of $\partial_{t}$ on $(\mathbf{C}\{t\} / \mathbf{C}[t]) \omega_{0}$ is bijective so that the morphism induced by $\mathcal{H}^{1} \beta$ is bijective.

We now calculate the cohomology sheaves of

$$
\underset{p}{\lim }\left(\underset{q}{\lim }\left(G_{p} / G_{q}\right) \mathcal{K}_{f}^{\bullet}\left[\partial_{t}^{-1}\right]\right)
$$

by using the Mittag-Leffler condition. We first calculate the projective limit for $q$ with $p$ fixed. Here we may assume that $p=0$ by using the action $\partial_{t}$. For $k^{\prime}=k+i>k>0$, we have the commutative diagrams

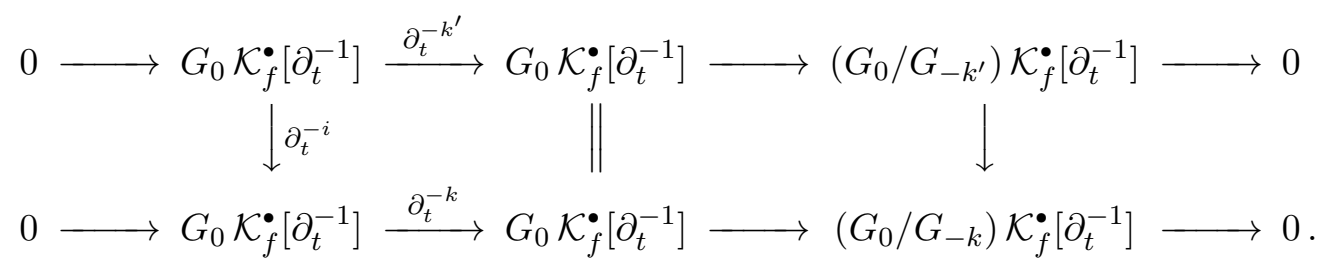

Combining this with (1.5.4) we get short exact sequences of projective systems for $j \in \mathbf{Z}$ :

$$
0 \rightarrow\left\{\widetilde{\mathcal{H}}^{j} / \partial_{t}^{-k} \widetilde{\mathcal{H}}^{j}\right\}_{k} \rightarrow\left\{\mathcal{H}^{j}\left(\left(G_{0} / G_{-k}\right) \mathcal{K}_{f}^{\bullet}\left[\partial_{t}^{-1}\right]\right)\right\}_{k} \rightarrow\left\{\operatorname{Ker}\left(\partial_{t}^{-k} \mid \widetilde{\mathcal{H}}^{j+1}\right)\right\}_{k} \rightarrow 0,
$$

where the transition morphisms of the first and last projective systems are induced by the identity on $\widetilde{\mathcal{H}}^{j}$ and $\partial_{t}^{-\left(k^{\prime}-k\right)}$ on $\widetilde{\mathcal{H}}^{j+1}$, respectively. By the middle property of (1.4.5), we see that the projective limit of the last projective system vanishes. So for $j \in \mathbf{Z}$ we get isomorphisms

$$
\lim _{k} \mathcal{H}^{j}\left(\left(G_{0} / G_{-k}\right) \mathcal{K}_{f}^{\bullet}\left[\partial_{t}^{-1}\right]\right)=\widehat{\mathcal{H}}_{f}^{j} \quad \text { with } \quad \widehat{\mathcal{H}}_{f}^{j}:={\underset{l}{k}}_{\lim _{f}} \widetilde{\mathcal{H}}_{f}^{j} / \partial_{t}^{-k} \widetilde{\mathcal{H}}_{f}^{j} \text {. }
$$

Moreover, the Mittag-Leffler condition is satisfied for $\left\{\mathcal{H}^{j}\left(\left(G_{0} / G_{-k}\right) \mathcal{K}_{f}^{\bullet}\left[\partial_{t}^{-1}\right]\right)\right\}_{k}$ by again using the middle property of (1.4.5) together with the snake lemma applied to the transition morphisms between the above short exact sequences of projective systems. So the projective limit commutes with the cohomology. 


\section{Sabbah And M. Saito}

We have to calculate the inductive limit of the projective limits. Here the inductive limit always commutes with the cohomology sheaf functor. The morphisms of the inductive system of the cohomology sheaves are induced by the inclusions

$$
G_{p} \mathcal{K}_{f}^{\bullet}\left[\partial_{t}^{-1}\right] \hookrightarrow G_{p+i} \mathcal{K}_{f}^{\bullet}\left[\partial_{t}^{-1}\right]
$$

which are identified with the morphisms

$$
\partial_{t}^{-i}: G_{0} \mathcal{K}_{f}^{\bullet}\left[\partial_{t}^{-1}\right] \hookrightarrow G_{0} \mathcal{K}_{f}^{\bullet}\left[\partial_{t}^{-1}\right]
$$

under the identification

$$
\partial_{t}^{p}: G_{0} \mathcal{K}_{f}^{\bullet}\left[\partial_{t}^{-1}\right] \stackrel{\sim}{\longrightarrow} G_{p} \mathcal{K}_{f}^{\bullet}\left[\partial_{t}^{-1}\right]
$$

So (1.5.1) follows from (1.5.5) since the inductive limit of the inductive system $\left\{\widehat{\mathcal{H}}_{f}^{j}\right\}_{k}$ with transition morphisms defined by $\partial_{t}^{-\left(k^{\prime}-k\right)}$ is the localization of $\widehat{\mathcal{H}}_{f}^{j}$ by $\partial_{t}^{-1}$. This completes the proof of Proposition 2.

\subsection{Proof of Theorem 2}

The assertion follows from Proposition 2 using (1.2.3) and (1.3.2).

\subsection{Remarks}

(i) The formal Brieskorn modules and Gauss-Manin systems defined by the $\partial_{t}^{-1}$-adic completion were used in [Sai88a] for hypersurface isolated singularities with non-degenerate Newton polygons where the Mittag-Leffler condition was also used. See also $\S 3.5$ below.

(ii) If $f^{-1}(0)$ is a divisor with normal crossings, the Brieskorn modules are $\partial_{t}^{-1}$-torsion-free, and the filtration $G$ gives the filtration $V$ of Kashiwara and Malgrange (indexed by $\mathbf{Z}$ ) by using an argument similar to [Ste76], (1.13), see also [Sai83]. It is possible to prove Proposition 2 in the normal crossing case by using this together with $\S 2.3$ below.

\subsection{Commutativity of inductive limit and global cohomology}

It is well known that the global cohomology functor does not necessarily commute with inductive limit nor with $((u))$ in the noncompact case. For instance, Cartan's Theorem B does not hold for quasi-coherent sheaves, see for example [Sai88b], 2.3.8. So we give here a sufficient condition for their commutativity.

Let $\mathcal{F}_{\lambda}^{\bullet}$ be an inductive system of complexes of sheaves on a topological space $X$ indexed by a directed set $\Lambda$. (In this paper, inductive systems are always indexed by directed sets.) We have the canonical flasque resolutions

$$
\mathcal{F}_{\lambda}^{\bullet} \rightarrow \mathcal{I}^{\bullet} \mathcal{F}_{\lambda}^{\bullet}, \quad \mathcal{F}^{\bullet} \rightarrow \mathcal{I}^{\bullet} \mathcal{F}^{\bullet} \quad \text { with } \mathcal{F}^{\bullet}:=\underset{\lambda}{\lim _{\lambda}} \mathcal{F}_{\lambda}^{\bullet},
$$

where $\mathcal{I}^{\bullet}$ denotes the canonical flasque resolution of Godement using the discontinuous sections. By the property of inductive limit, there is a canonical morphism

$$
\underset{\lambda}{\lim _{\lambda}} \mathbf{R} \Gamma\left(X, \mathcal{F}_{\lambda}^{\bullet}\right)\left(:=\underset{\lambda}{\lim } \Gamma\left(X, \mathcal{I}^{\bullet} \mathcal{F}_{\lambda}^{\bullet}\right)\right) \rightarrow \mathbf{R} \Gamma\left(X, \mathcal{F}^{\bullet}\right)\left(:=\Gamma\left(X, \mathcal{I}^{\bullet} \mathcal{F}^{\bullet}\right)\right)
$$

Assume that $X$ has a compactification $Y$ with a stratification compatible with $X$ and satisfying the local triviality condition using the local cone structure along each stratum. More precisely, there is an increasing sequence of closed subspaces $Y_{k}(k \geqslant-1)$ with $Y_{-1}=\varnothing$, $Y_{d}=Y(d \gg 0)$, and for any $x \in Y_{d} \backslash Y_{d-1}$ with $d \geqslant 0$, there is an open neighborhood $U_{x}$ of $x$ in $Y$ 


\section{KONTSEVICH's CONJECTURE}

together with a compact topological space $L_{x}$ having an increasing sequence of closed subspaces $\left(L_{x}\right)_{k}(k \geqslant-1)$ with $\left(L_{x}\right)_{-1}=\varnothing$ and such that there is a homeomorphism $U_{x} \cong \mathbf{R}^{d} \times C\left(L_{x}\right)$ inducing $Y_{k} \cap U_{x} \cong \mathbf{R}^{d} \times C\left(\left(L_{x}\right)_{k-d-1}\right)$ for any $k \geqslant d$, see for example [GM80].

Set $Y_{k}^{o}:=Y_{k} \backslash Y_{k-1}$. Assume furthermore that the following condition is satisfied:

$$
\left.\mathcal{H}^{i} \mathcal{F}_{\lambda}^{\bullet}\right|_{Y_{k}^{o}} \text { are locally constant for any } \lambda, k, i \text {. }
$$

We can then show that (1.8.1) is an quasi-isomorphism.

Let $j: X \hookrightarrow Y$ denote the inclusion. We first prove the canonical quasi-isomorphism

$$
\underset{\lambda}{\lim } \mathbf{R} j_{*} \mathcal{F}_{\lambda}^{\bullet}\left(:=\underset{\lambda}{\lim } j_{*} \mathcal{I}^{\bullet} \mathcal{F}_{\lambda}^{\bullet}\right) \stackrel{\sim}{\longrightarrow} \mathbf{R} j_{*} \mathcal{F}^{\bullet}\left(:=j_{*} \mathcal{I}^{\bullet} \mathcal{F}^{\bullet}\right) \text {. }
$$

Since the inductive limit commutes with $\mathcal{H}^{i}$, we see that condition $(\mathrm{C})$ also holds for $\mathcal{F}^{\bullet}$. Using the local cone structure along each stratum, condition (C) then holds for $\mathbf{R} j_{*} \mathcal{F}_{\lambda}^{\bullet}$ and $\mathbf{R} j_{*} \mathcal{F}^{\bullet}$. Consider the open inclusions

$$
j_{k}: X \cup\left(Y \backslash Y_{k}\right) \hookrightarrow X \cup\left(Y \backslash Y_{k-1}\right) .
$$

Let $j_{k}^{\prime}$ be the composition of the $j_{i}$ for $i \geqslant k$. Condition $(C)$ for $\mathbf{R}\left(j_{k+1}^{\prime}\right)_{*} \mathcal{F}_{\lambda}^{\bullet}$ implies the canonical isomorphisms for $x \in Y_{k}^{0}$

$$
H^{i}\left(U_{x} \backslash Y_{k},\left.\mathbf{R}\left(j_{k+1}^{\prime}\right)_{*} \mathcal{F}_{\lambda}^{\cdot}\right|_{U_{x} \backslash Y_{k}}\right) \stackrel{\sim}{\longrightarrow} H^{i}\left(L_{x},\left.\mathbf{R}\left(j_{k+1}^{\prime}\right)_{*} \mathcal{F}_{\lambda}^{\bullet}\right|_{L_{x}}\right),
$$

where $L_{x}$ is identified with $\{0\} \times\left(\left\{\frac{1}{2}\right\} \times L_{x}\right) \subset \mathbf{R}^{d} \times C\left(L_{x}\right) \cong U_{x}$. We have the same for $\mathbf{R}\left(j_{k+1}^{\prime}\right)_{*} \mathcal{F}^{\bullet}$. So we can prove (1.8.2) at each stratum $Y_{k}^{o}$ by decreasing induction on $k$ using the following well-known property:

$$
\text { Inductive limit commutes with the global cohomology on compact spaces. }
$$

By (1.8.2) and (1.8.4), we thus get the canonical isomorphism

$$
\underset{\lambda}{\lim } \mathbf{R} \Gamma\left(X, \mathcal{F}_{\lambda}^{\bullet}\right) \stackrel{\sim}{\longrightarrow} \mathbf{R} \Gamma\left(X, \mathcal{F}^{\bullet}\right) .
$$

\section{Formal microlocalization}

In this section we recall some basics of formal microlocalization and prove Proposition 4 by reducing to the normal crossing case.

\subsection{Differential complexes and formal microlocalization}

In this subsection $X$ is either a smooth complex algebraic variety or a complex manifold. By [Sai89], Prop. 1.8, there is an equivalence of categories

$$
\begin{array}{ll} 
& \operatorname{DR}_{X}^{-1}: D^{b}\left(\mathcal{O}_{X}, \text { Diff }\right) \stackrel{\sim}{\longrightarrow} D^{b}\left(\mathcal{D}_{X}\right), \\
\text { with } & \operatorname{DR}_{X}^{-1}(\mathcal{L}):=\mathcal{L} \otimes_{\mathcal{O}_{X}} \mathcal{D}_{X} \text { for } \mathcal{O}_{X} \text {-modules } \mathcal{L},
\end{array}
$$

where $D^{b}\left(\mathcal{D}_{X}\right)$ is the bounded derived category of right $\mathcal{D}_{X}$-modules, and $D^{b}\left(\mathcal{O}_{X}\right.$, Diff $)$ is the derived category of bounded complexes whose components are $\mathcal{O}_{X}$-modules and whose differentials are differential morphisms in the sense of loc. cit. More precisely, the differential morphisms are defined so that for $\mathcal{O}_{X}$-modules $\mathcal{L}, \mathcal{L}^{\prime}$, we have

$$
\mathcal{H o m}_{\text {Diff }}\left(\mathcal{L}, \mathcal{L}^{\prime}\right)=\mathcal{H} m_{\mathcal{D}_{X}}\left(\operatorname{DR}_{X}^{-1}(\mathcal{L}), \mathrm{DR}_{X}^{-1}\left(\mathcal{L}^{\prime}\right)\right),
$$




\section{Sabbah and M. Saito}

and the derived category is defined by inverting $\mathcal{D}$-quasi-isomorphisms (which are, by definition, morphisms whose mapping cones are $\mathcal{D}$-acyclic, where the last condition is defined by the acyclicity of the image by the functor $\mathrm{DR}_{X}^{-1}$, see loc. cit.) Note that for $\mathcal{L}^{\bullet} \in D^{b}\left(\mathcal{O}_{X}\right.$, Diff) and $\mathcal{M}^{\bullet} \in D^{b}\left(\mathcal{D}_{X}\right)$, there are canonical $\mathcal{D}$-quasi-isomorphisms and quasi-isomorphisms

$$
\operatorname{DR}_{X}\left(\operatorname{DR}_{X}^{-1}\left(\mathcal{L}^{\bullet}\right)\right) \stackrel{\sim}{\longrightarrow} \mathcal{L}^{\bullet}, \quad \operatorname{DR}_{X}^{-1}\left(\operatorname{DR}_{X}\left(\mathcal{M}^{\bullet}\right)\right) \stackrel{\sim}{\longrightarrow} \mathcal{M}^{\bullet},
$$

where $\operatorname{DR}_{X}(\mathcal{M})$ is the (shifted) de Rham complex for right $\mathcal{D}_{X}$-modules $\mathcal{M}$.

Let $D_{r h}^{b}\left(\mathcal{D}_{X}\right)$ denote the full subcategory of $D^{b}\left(\mathcal{D}_{X}\right)$ consisting of the $\mathcal{M}^{\bullet}$ with $\mathcal{H}^{j} \mathcal{M}^{\bullet}$ regular holonomic over $\mathcal{D}_{X}$. Let $D_{r h}^{b}\left(\mathcal{O}_{X}\right.$, Diff $)$ be the full subcategory of $D^{b}\left(\mathcal{O}_{X}\right.$, Diff $)$ consisting of the $\mathcal{L}^{\bullet}$ with $\operatorname{DR}_{X}^{-1}\left(\mathcal{L}^{\bullet}\right) \in D_{r h}^{b}\left(\mathcal{D}_{X}\right)$. We have an equivalence of categories

$$
\mathrm{DR}_{X}^{-1}: D_{r h}^{b}\left(\mathcal{O}_{X}, \text { Diff }\right) \stackrel{\sim}{\longrightarrow} D_{r h}^{b}\left(\mathcal{D}_{X}\right) .
$$

By (2.1.1), any $\mathcal{L}^{\bullet} \in D_{r h}^{b}\left(\mathcal{O}_{X}\right.$, Diff $)$ is represented by $\operatorname{DR}_{X}\left(\operatorname{DR}_{X}^{-1}\left(\mathcal{L}^{\bullet}\right)\right)$, and, moreover, the $\operatorname{DR}_{X}(\mathcal{M})$ for $\mathcal{M} \in M_{r h}\left(\mathcal{D}_{X}\right)$ are $\mathcal{D}$-quasi-isomorphic to subcomplexes $F_{p} \mathrm{DR}_{X}(\mathcal{M})$ (whose components are coherent over $\left.\mathcal{O}_{X}\right)$ for $p \gg 0$ locally on $X$, where $F$ is induced by a good filtration on $\mathcal{M}$ locally defined on $X$. By the functor $\mathrm{DR}^{-1}$, the direct image of $\mathcal{D}$-modules is compatible with that of differential complexes, and the latter is defined by the sheaf-theoretic direct image, see loc. cit., Prop. 3.3.

Let $f$ be an algebraic or holomorphic function on $X$. Set $S:=\mathbf{C}$. We have the graph embedding

$$
i_{f}: X \hookrightarrow \mathcal{X}:=X \times S .
$$

We define the formal microlocalization $\mathcal{L}^{\bullet}((u))^{f}$ of $\mathcal{L}^{\bullet} \in D_{\text {rh }}^{b}\left(\mathcal{O}_{X}\right.$, Diff $)$ as follows.

Consider first $\mathrm{DR}_{\mathcal{X}}^{-1} \mathcal{L}^{\bullet}$, where $\mathcal{L}^{\bullet}$ is identified with the direct image by $i_{f}$. It is a complex of right $\mathcal{D}_{\mathcal{X}}$-modules whose $j$ th component is $\mathcal{L}^{j} \otimes_{\mathcal{O}_{\mathcal{X}}} \mathcal{D}_{\mathcal{X}}$. Take the tensor product over $\mathcal{D}_{\mathcal{X}}$ with

$$
\mathcal{D}_{\mathcal{X} \rightarrow S}:=\mathcal{O}_{\mathcal{X}} \otimes_{p r_{2}^{-1} \mathcal{O}_{S}} p r_{2}^{-1} \mathcal{D}_{S}
$$

It has a structure of a complex of right $\mathrm{pr}_{2}^{-1} \mathcal{D}_{S}$-modules. We convert it to a complex of left $p r_{2}^{-1} \mathcal{D}_{S}$-modules by using the involution * of $\mathcal{D}_{S}$ such that

$$
(P Q)^{*}=Q^{*} P^{*}, \quad g^{*}=g\left(g \in \mathcal{O}_{S}\right), \quad \partial_{t}^{*}=-\partial_{t} .
$$

We thus get the first of the following three complexes

$$
\mathcal{L}^{\bullet}\left[\partial_{t}\right]^{f}, \quad \mathcal{L}^{\bullet}\left[\partial_{t}, \partial_{t}^{-1}\right]^{f}, \quad \mathcal{L}^{\bullet}\left(\left(\partial_{t}^{-1}\right)\right)^{f} .
$$

The second complex is then obtained by taking the localization of the first by $\partial_{t}$, and the third by the tensor product of the second with $\mathcal{O}_{X}\left(\left(\partial_{t}^{-1}\right)\right.$ ) over $\mathcal{O}_{X}\left[\partial_{t}, \partial_{t}^{-1}\right]$ (which is well defined since $\mathcal{O}_{X}\left(\left(\partial_{t}^{-1}\right)\right)$ is flat over $\left.\mathcal{O}_{X}\left[\partial_{t}, \partial_{t}^{-1}\right]\right)$. Note that the above definition using the tensor product is reasonable only in the case where the $\mathcal{H}^{j} \mathrm{DR}^{-1}\left(\mathcal{L}^{\bullet}\right)$ are coherent (for example regular holonomic) over $\mathcal{D}_{X}$, see the remark after (2.1.2).

The upperscript ${ }^{f}$ means that the differential and the action of $t$ are twisted by using $f$ as in the introduction, and $\mathcal{L}^{\bullet}\left(\left(\partial_{t}^{-1}\right)\right)^{f}$ may be understood as an abbreviation of $\mathcal{L}^{\bullet}\left(\left(\partial_{t}^{-1}\right)\right) \delta(f-t)$ where $\delta(f-t)$ is the delta function satisfying the relations

$$
t \delta(f-t)=f \delta(f-t), \quad \xi \delta(f-t)=-(\xi f) \delta(f-t) \quad \text { for } \xi \in \Theta_{X} .
$$

If $\mathcal{L}^{\bullet}=\mathrm{DR}_{X}(\mathcal{M})$ for a locally finite free $\mathcal{O}_{X}$-module $\mathcal{M}$ with an integrable connection, we have a canonical isomorphism

$$
\mathcal{L}^{\bullet}\left(\left(\partial_{t}^{-1}\right)\right)^{f}=\mathrm{DR}_{X}\left(\mathcal{M}\left(\left(\partial_{t}^{-1}\right)\right)^{f}\right) .
$$




\section{KONTSEVICH'S CONJECTURE}

Moreover, the construction of $\mathcal{L}^{\bullet}\left(\left(\partial_{t}^{-1}\right)\right)^{f}$ is compatible with the direct image by a proper morphism $\pi: X^{\prime} \rightarrow X$, that is, for a differential complex $\mathcal{L}^{\bullet}$ on $X^{\prime}$, we have

$$
\mathbf{R} \pi_{*}\left(\mathcal{L}^{\bullet}\left(\left(\partial_{t}^{-1}\right)\right)^{\pi^{*} f}\right)=\left(\mathbf{R} \pi_{*} \mathcal{L}^{\bullet}\right)\left(\left(\partial_{t}^{-1}\right)\right)^{f},
$$

where $\mathbf{R} \pi_{*}$ is defined by the sheaf-theoretic direct image. Here we may assume that each component of the complexes is $\pi_{*}$-acyclic by taking the canonical flasque resolution of Godement. It is then enough to show the compatibility with the differential of the complexes where the formal microlocalization can be replaced with the algebraic one by the above argument. So the assertion is shown by using the direct image of induced $\mathcal{D}$-modules as in the proof of [Sai89], Prop. 3.3.

We will also consider the case where the divisor at infinity $D$ is given in $\bar{X}$ (especially in the analytic case). In this case, we similarly define $D_{r h}^{b}\left(\mathcal{O}_{\bar{X}}(* D)\right.$, Diff) by assuming that the $\overline{\mathcal{L}}^{j}$ are $\mathcal{O}_{\bar{X}}(* D)$-modules and that the $\mathcal{H}^{j} \mathrm{DR}_{X}^{-1}\left(\overline{\mathcal{L}}^{\bullet}\right)$ are regular holonomic over $\mathcal{D}_{\bar{X}}$, and then define the formal microlocalization $\overline{\mathcal{L}}^{\bullet}\left(\left(\partial_{t}^{-1}\right)\right)^{f}$ by using the tensor product with $\mathcal{O}_{\bar{X}}(* D)\left(\left(\partial_{t}^{-1}\right)\right)^{f}$ over $\mathcal{O}_{\bar{X}}(* D)$ instead of the one with $\mathcal{O}_{\bar{X}}\left(\left(\partial_{t}^{-1}\right)\right)^{f}$ over $\mathcal{O}_{\bar{X}}$. (For the difference between these, see Example 2.2 below.) We also have the compatibility with the direct image as above by setting $D^{\prime}:=\bar{\pi}^{-1}(D)$ for a proper morphism $\bar{\pi}: \bar{X}^{\prime} \rightarrow \bar{X}$. These will be used at the end of $\S 2.3$ below.

\subsection{Example}

Set $X:=\mathbf{C}, E:=\{0\}$, and also $\bar{X}:=\mathbf{C}, D:=\{0\}$. Let $x$ be the coordinate of $\mathbf{C}$. Set

$$
\mathcal{L}^{\bullet}:=C\left(x \partial_{x}: \mathcal{O}_{X} \rightarrow \mathcal{O}_{X}\right), \quad \overline{\mathcal{L}} \bullet:=C\left(x \partial_{x}: \mathcal{O}_{\bar{X}}(* D) \rightarrow \mathcal{O}_{\bar{X}}(* D)\right) .
$$

We have

$$
\mathcal{D}_{X} / \mathcal{D}_{X} \partial_{x} x=\mathcal{O}_{X}\left[x^{-1}\right]=\mathcal{O}_{X}(* E)
$$

and there are canonical quasi-isomorphisms

$$
\operatorname{DR}_{X}^{-1}\left(\mathcal{L}^{\bullet}\right) \stackrel{\sim}{\longrightarrow} \mathcal{O}_{X}(* E), \quad \operatorname{DR}_{\bar{X}}^{-1}\left(\overline{\mathcal{L}}^{\bullet}\right) \stackrel{\sim}{\longrightarrow} \mathcal{O}_{\bar{X}}(* D),
$$

where the last one follows from the first by using the localization. (The difference between $x \partial_{x}$ and $\partial_{x} x$ comes from the involution ${ }^{*}$ of $\mathcal{D}_{X}$ in (2.1.3) for the transformation between left and right $\mathcal{D}$-modules.)

However, setting $f=x$, we can easily show by a direct calculation that

$$
\mathcal{L}^{\bullet}\left(\left(\partial_{t}^{-1}\right)\right)^{f} \stackrel{\sim}{\longrightarrow} \mathbf{C}\left(\left(\partial_{t}^{-1}\right)\right)_{0}, \quad \overline{\mathcal{L}}^{\bullet}\left(\left(\partial_{t}^{-1}\right)\right)^{f}=0
$$

where $\mathbf{C}\left(\left(\partial_{t}^{-1}\right)\right)_{0}$ is the sheaf supported at the origin with stalk $\mathbf{C}\left(\left(\partial_{t}^{-1}\right)\right)$.

\subsection{Reduction of Proposition 4 to the normal crossing case}

Let $\bar{\pi}: \bar{X}^{\prime} \rightarrow \bar{X}$ be a proper birational morphism of smooth complex algebraic varieties such that the union of the pullback $D^{\prime}$ of $D$ and a finite number of fibers $\bar{X}_{c}^{\prime}(c \in \Sigma)$ is a divisor with simple normal crossings, where $\Sigma \subset \mathbf{P}^{1}$ is a finite set containing $\infty$, and moreover the restriction of $\left(\bar{X}^{\prime}, D^{\prime}\right)$ over $S^{\prime}:=S \backslash \Sigma$ is a divisor with simple normal crossings over $S^{\prime}$. The latter condition means that the restriction over $S^{\prime}$ of any intersection of irreducible components of $D^{\prime}$ is smooth over $S^{\prime}$. Set $X^{\prime}:=\bar{\pi}^{-1}(X), \pi:=\left.\bar{\pi}\right|_{X^{\prime}}: X^{\prime} \rightarrow X$, and $\mathcal{M}^{\prime}:=\pi^{*} \mathcal{M}$. There is a canonical morphism of differential complexes

$$
\pi^{\#}: \mathrm{DR}_{X}(\mathcal{M}) \rightarrow \mathbf{R} \pi_{*} \mathrm{DR}_{X^{\prime}}\left(\mathcal{M}^{\prime}\right) .
$$


Applying this to the dual $\mathcal{M}^{*}:=\mathcal{H}^{\circ} m_{\mathcal{O}_{X}}\left(\mathcal{M}, \mathcal{O}_{X}\right)$ and using the duality for the direct images of differential complexes (see [Sai89], Th. 3.11), we get

$$
\pi_{\#}: \mathbf{R} \pi_{*} \mathrm{DR}_{X^{\prime}}\left(\mathcal{M}^{\prime}\right) \rightarrow \mathrm{DR}_{X}(\mathcal{M}) .
$$

Since the composition $\pi_{\#} \circ \pi^{\#}$ is the identity on $\operatorname{DR}_{X}(\mathcal{M})$, we get an isomorphism

$$
\mathbf{R} \pi_{*} \mathrm{DR}_{X^{\prime}}\left(\mathcal{M}^{\prime}\right) \cong \operatorname{DR}_{X}(\mathcal{M}) \oplus \text { Cone } \pi^{\#} \quad \text { in } D^{b}\left(\mathcal{O}_{X}, \text { Diff }\right)^{f},
$$

together with

$$
\text { Cone } \pi^{\#} \cong \text { Cone } \pi_{\#}[-1] \text {. }
$$

Indeed, these follow from the octahedral axiom of the triangulated category (applied to the composition of $\pi^{\#}$ and $\pi_{\#}$ ).

Let $j^{\prime}: X^{\prime} \hookrightarrow \bar{X}^{\prime}$ denote the inclusion. Set $\overline{\mathcal{M}}^{\prime}:=j_{*} \mathcal{M}^{\prime}$. By (2.3.3) and GAGA, we have

$$
\mathrm{DR}_{\bar{X}^{\text {an }}}\left(\overline{\mathcal{M}}^{\text {an }}\right) \text { is a direct factor of } \mathbf{R} \bar{\pi}_{*} \mathrm{DR}_{\bar{X}^{\prime \text { an }}}\left(\overline{\mathcal{M}}^{\prime \text { an }}\right) \text {. }
$$

Set $f^{\prime}:=\pi^{*} f$. By (2.1.5) together with the remark after it, we then get

$$
\operatorname{DR}_{\bar{X}^{\text {an }}}\left(\overline{\mathcal{M}}^{\text {an }}\right)\left(\left(\partial_{t}^{-1}\right)\right)^{f} \text { is a direct factor of } \mathbf{R} \bar{\pi}_{*}\left(\operatorname{DR}_{\bar{X}^{\prime a n}}\left(\overline{\mathcal{M}}^{\prime \text { an }}\right)\left(\left(\partial_{t}^{-1}\right)\right)^{f^{\prime}}\right) \text {. }
$$

Here we use the remark at the end of $\S 2.1$. Thus Proposition 4 is reduced to the case where the union of $D:=\bar{X} \backslash X$ and the fibers $\bar{X}_{c}(c \in \Sigma)$ is a divisor with normal crossings.

\subsection{Proof of Proposition 4 in the normal crossing case}

Since the assertion is local on $\bar{X}$, we may restrict to an open neighborhood in $\bar{X}$ of a point of $\bar{X}_{c} \backslash X_{c}$, where we may assume that $c=0$ or $\infty$ (by replacing $f$ with $f-c$ when $c \neq \infty$ ). By $\S 2.3$ we may assume that $X, \bar{X}$ are complex manifolds of dimension $n$ such that the union of $D:=\bar{X} \backslash X$ and $f^{-1}(0)$ is a divisor with normal crossings on $\bar{X}$. We take a neighborhood of $0 \in \bar{X}_{c}$ in $\bar{X}$ of the form

$$
U_{\varepsilon}=\Delta_{\varepsilon}^{n} \subset \bar{X}
$$

where $\Delta_{\varepsilon}$ is an open disk of radius $\varepsilon$. We may assume that there are subsets $J, J^{\prime} \subset\{1, \ldots, n\}$ such that

$$
U_{\varepsilon}^{\prime}:=U_{\varepsilon} \cap X=\left\{\prod_{i \in J^{\prime}} x_{i} \neq 0\right\}, \quad f=\prod_{i \in J} x_{i}^{e_{i}},
$$

replacing $U_{\varepsilon}$ and $\varepsilon$ if necessary, where $x_{1}, \ldots, x_{n}$ are the coordinates of the polydisk $\Delta_{\varepsilon}^{n}$. Note that $e_{i} \leqslant-1$ and $J^{\prime} \supset J$ if $c=\infty$, and $e_{i} \geqslant 1$ if $c=0$. In the inductive argument below, we have to also treat the case $f=0$ where we set $J=\varnothing$. We assume that $J \neq \varnothing$ (that is, that $f \neq 0$ ) for the moment until the proof for the case $J=\varnothing$ will be explained.

We first treat the case $c=0$. Since the assertion is local on $\bar{X}$, we may replace $\bar{X}, X$ with $U_{\varepsilon}, U_{\varepsilon}^{\prime}$ respectively. We will denote by $\overline{\mathcal{M}}, \mathcal{M}$ their restrictions to $U_{\varepsilon}, U_{\varepsilon}^{\prime}$ in this subsection. The assertion is reduced to the case $\operatorname{rank} \mathcal{M}=1$ by using a filtration on $\mathcal{M}$ since the local monodromies are commutative in the normal crossing case. Then there is a generator $m$ of $\overline{\mathcal{M}}$ annihilated by the differential operators

$$
\xi_{i}:= \begin{cases}\partial_{x_{i}} x_{i}-\alpha_{i} & \text { if } i \in J^{\prime}, \\ \partial_{x_{i}} & \text { if } i \notin J^{\prime},\end{cases}
$$

where $\alpha_{i} \in \mathbf{C}$, and we have $\overline{\mathcal{M}}=\mathcal{D}_{U_{\varepsilon}} / \mathcal{J}$ with $\mathcal{J}$ generated by the above differential operators.

Replacing $\xi_{i}=\partial_{x_{i}}$ with $\xi_{i}^{\prime}:=\partial_{x_{i}} x_{i}$ for $i \in J \backslash J^{\prime}$, we get a left ideal $\mathcal{J}^{\prime}$ of $\mathcal{D}_{U_{\varepsilon}}$ generated by 


\section{KONTSEVICH'S CONJECTURE}

the $\xi_{i}^{\prime}$, where $\xi_{i}^{\prime}:=\xi_{i}$ if $i \notin J \backslash J^{\prime}$. Then there is an injection

$$
\overline{\mathcal{M}} \hookrightarrow \overline{\mathcal{M}}^{\prime}:=\mathcal{D}_{U_{\varepsilon}} / \mathcal{J}^{\prime}
$$

defined by the multiplication by $\prod_{i \in J \backslash J^{\prime}} x_{i}$. Moreover, there is a finite increasing filtration $W$ on $\overline{\mathcal{M}}^{\prime}$ such that

$$
\operatorname{Gr}_{k}^{W} \overline{\mathcal{M}}^{\prime}=\bigoplus_{I \subset J \backslash J^{\prime},|I|=k} \overline{\mathcal{M}}_{I}
$$

where the $\overline{\mathcal{M}}_{I}$ are $\mathcal{D}_{U_{\varepsilon}}$-modules of the same type as $\overline{\mathcal{M}}$ (up to the direct image as $\mathcal{D}$-modules by closed immersions), and are supported on

$$
U_{\varepsilon, I}:=\bigcap_{i \in I}\left\{x_{i}=0\right\} \subset U_{\varepsilon} .
$$

If $|I|=k>0$, then $U_{\varepsilon, I}$ is contained in $f^{-1}(0)$. The assertion in this case is reduced to the case $f=0$ (that is, $J=\varnothing$ ) by using differential complexes as in $\S 2.1$, and can be proved by using the inductive argument in this subsection. We may thus replace $\overline{\mathcal{M}}$ with $\overline{\mathcal{M}}^{\prime}$.

Since the $\xi_{i}^{\prime}$ give a free resolution of $\overline{\mathcal{M}}^{\prime}$, we get an isomorphism

$$
\operatorname{DR}_{U_{\varepsilon}}\left(\overline{\mathcal{M}}^{\prime}\right)=K^{\bullet}\left(\mathcal{O}_{U_{\varepsilon}} ; \xi_{i}^{* *}\right)[n],
$$

where the right-hand side is the (shifted) Koszul complex associated with the mutually commuting operators $\xi_{i}^{\prime *}$ with ${ }^{*}$ the involution of $\mathcal{D}_{U_{\varepsilon}}$ as in (2.1.3). Indeed, the right-hand side is actually $K^{\bullet}\left(\Omega_{U_{\varepsilon}}^{n} ; \xi_{i}^{\prime}\right)[n]$. Here we may replace $\xi_{i}^{*}$ with $-\xi_{i}^{\prime *}$.

Let $g:=\prod_{i \in J^{\prime}} x_{i}$ so that $U_{\varepsilon} \backslash U_{\varepsilon}^{\prime}=g^{-1}(0)$. Set

$$
A_{\varepsilon}:=\Gamma\left(U_{\varepsilon}, \mathcal{O}_{U_{\varepsilon}}\right)\left[\frac{1}{g}\right], \quad A_{\varepsilon}^{\prime}:=\Gamma\left(U_{\varepsilon}^{\prime}, \mathcal{O}_{U_{\varepsilon}^{\prime}}\right) \quad A_{\varepsilon}^{\prime \prime}=A_{\varepsilon}^{\prime} / A_{\varepsilon} .
$$

By the above calculation of the de Rham complex $\operatorname{DR}_{U_{\varepsilon}}\left(\overline{\mathcal{M}}^{\prime}\right)$ using the Koszul complex of the operators $-\xi_{i}^{\prime *}$, its twisted de Rham complex is given by the Koszul complex of the following operators:

More precisely, let

$$
\widetilde{\xi}_{i}:= \begin{cases}x_{i} \partial_{x_{i}}+\alpha_{i}+e_{i} f \partial_{t} & \text { if } i \in J \cap J^{\prime} \\ x_{i} \partial_{x_{i}}+e_{i} f \partial_{t} & \text { if } i \in J \backslash J^{\prime} \\ x_{i} \partial_{x_{i}}+\alpha_{i} & \text { if } i \in J^{\prime} \backslash J \\ \partial_{x_{i}} & \text { if } i \notin J \cup J^{\prime}\end{cases}
$$

$$
K^{\bullet}\left(A_{\varepsilon}\left(\left(\partial_{t}^{-1}\right)\right), \widetilde{\xi}_{i}\right), \quad K^{\bullet}\left(A_{\varepsilon}^{\prime}\left(\left(\partial_{t}^{-1}\right)\right), \widetilde{\xi}_{i}\right), \quad K^{\bullet}\left(A_{\varepsilon}^{\prime \prime}\left(\left(\partial_{t}^{-1}\right)\right), \widetilde{\xi}_{i}\right)
$$

denote the Koszul complex associated with the mutually commuting differential operators $\widetilde{\xi}_{i}$ on $A_{\varepsilon}\left(\left(\partial_{t}^{-1}\right)\right), A_{\varepsilon}^{\prime}\left(\left(\partial_{t}^{-1}\right)\right)$ and $A_{\varepsilon}^{\prime \prime}\left(\left(\partial_{t}^{-1}\right)\right)$, respectively. For the proof of Proposition 4, we have to show the canonical quasi-isomorphism

$$
\overline{\mathcal{M}}\left(\left(\partial_{t}^{-1}\right)\right)^{f} \otimes_{\mathcal{O}} \Omega_{U_{\varepsilon}}^{\bullet} \rightarrow \mathbf{R} j_{*}\left(\mathcal{M}\left(\left(\partial_{t}^{-1}\right)\right)^{f} \otimes \mathcal{O} \Omega_{U_{\varepsilon}^{\prime}}^{\bullet}\right) .
$$

We first show that the stalk at the origin of this morphism is identified with the inductive limit for $\varepsilon \rightarrow 0$ of the canonical morphism

$$
K^{\bullet}\left(A_{\varepsilon}\left(\left(\partial_{t}^{-1}\right)\right), \widetilde{\xi}_{i}\right) \rightarrow K^{\bullet}\left(A_{\varepsilon}^{\prime}\left(\left(\partial_{t}^{-1}\right)\right), \widetilde{\xi}_{i}\right) .
$$

Indeed, the assertion for $K^{\bullet}\left(A_{\varepsilon}\left(\left(\partial_{t}^{-1}\right)\right), \widetilde{\xi}_{i}\right)$ follows from the definition of the projective limit of sheaves. As for $K^{\bullet}\left(A_{\varepsilon}^{\prime}\left(\left(\partial_{t}^{-1}\right)\right), \widetilde{\xi}_{i}\right)$, set

$$
\widehat{\mathcal{K}}_{f}^{\prime \bullet}:=\mathcal{M}^{\prime}\left(\left(\partial_{t}^{-1}\right)\right)^{f} \otimes_{\mathcal{O}} \Omega_{U_{\varepsilon}^{\prime}}^{\bullet} \quad \text { with } \quad \mathcal{M}^{\prime}:=\left.\overline{\mathcal{M}}^{\prime}\right|_{U_{\varepsilon}^{\prime}}
$$




\section{SABbah And M. SAito}

where the differential is defined as in the introduction. We have isomorphisms of complexes

$$
K^{\bullet}\left(A_{\varepsilon}^{\prime}\left(\left(\partial_{t}^{-1}\right)\right), \widetilde{\xi}_{i}\right)=\Gamma\left(U_{\varepsilon}^{\prime}, \widehat{\mathcal{K}}_{f}^{\prime \bullet}\right)=\underset{p}{\lim } \Gamma\left(U_{\varepsilon}^{\prime}, G_{p} \widehat{\mathcal{K}}_{f}^{\prime \bullet}\right),
$$

where the filtration $G$ on $\widehat{\mathcal{K}}_{f}^{\prime \bullet}$ is defined in the same way as in $\S 1.5$. We moreover have a canonical quasi-isomorphism

$$
\Gamma\left(U_{\varepsilon}^{\prime}, G_{p} \widehat{\mathcal{K}}_{f}^{\prime \bullet}\right) \stackrel{\sim}{\longrightarrow} \mathbf{R} \Gamma\left(U_{\varepsilon}^{\prime}, G_{p} \widehat{\mathcal{K}}_{f}^{\prime \bullet}\right)
$$

since the infinite direct product of sheaves $\prod$ commutes with cohomology and $U_{\varepsilon}^{\prime}$ is Stein. We can show that their restrictions to the strata of the natural stratification of a divisor with normal crossings are locally constant by reducing to the case of Remark 1.7(ii) using the filtration $W$ on $\overline{\mathcal{M}}^{\prime}$ defined above. So we can apply $\S 1.8$ to get the commutativity of the inductive limit and $\mathbf{R} \Gamma\left(U_{\varepsilon}^{\prime}, *\right)$. Combining this with (2.4.2)-(2.4.3), we get the quasi-isomorphism

$$
K^{\bullet}\left(A_{\varepsilon}^{\prime}\left(\left(\partial_{t}^{-1}\right)\right), \widetilde{\xi}_{i}\right) \stackrel{\sim}{\longrightarrow} \mathbf{R} \Gamma\left(U_{\varepsilon}^{\prime}, \widehat{\mathcal{K}}_{f}^{\prime \bullet}\right) .
$$

The proof of Proposition 4 in the normal crossing case is thus reduced to the assertion that (2.4.1) is a quasi-isomorphism. Note that this is equivalent to the assertion that

$$
K^{\bullet}\left(A_{\varepsilon}^{\prime \prime}\left(\left(\partial_{t}^{-1}\right)\right), \widetilde{\xi}_{i}\right)=0 .
$$

Here we may assume that

$$
J \backslash J^{\prime} \neq \varnothing \text {, that is, } J \not \subset J^{\prime} .
$$

Indeed, if $J \subset J^{\prime}$, then the target of (2.4.1) vanishes, and we get the vanishing of the source by using the $\partial_{t}^{-1}$-adic filtration on $A_{\varepsilon}\left(\left(\partial_{t}^{-1}\right)\right)$ defined by $A_{\varepsilon} \llbracket \partial_{t}^{-1} \rrbracket \partial_{t}^{-k}$ for $k \in \mathbf{Z}$. Here the graded piece of $\widetilde{\xi}_{i}$ for $i \in J$ is given by $e_{i} f \partial_{t}$ and $f$ is invertible in $A_{\varepsilon}$ by the condition $J \subset J^{\prime}$. A similar argument applies to the case $c=\infty$.

For an element of $A_{\varepsilon}\left(\left(\partial_{t}^{-1}\right)\right)$ or $A_{\varepsilon}^{\prime}\left(\left(\partial_{t}^{-1}\right)\right)$, we have the Laurent expansion

$$
\sum_{\nu \in \mathbf{Z}^{n}} a_{\nu} x^{\nu} \text { with } a_{\nu} \in \mathbf{C},
$$

where the coefficients $a_{\nu}$ satisfy certain convergence conditions as is well known. Since

$$
\left(x_{i} \partial_{x_{i}}-\nu_{i}\right) x^{\nu}=0
$$

we see that $K^{\bullet}\left(A_{\varepsilon}\left(\left(\partial_{t}^{-1}\right)\right), \widetilde{\xi}_{i}\right), K^{\bullet}\left(A_{\varepsilon}^{\prime}\left(\left(\partial_{t}^{-1}\right)\right), \widetilde{\xi}_{i}\right)$ are acyclic when $\alpha_{i} \notin \mathbf{Z}$ for some $i \in J^{\prime} \backslash J$ (using the $n$-tuple complex structure of the Koszul complex). We may thus assume that

$$
\alpha_{i} \in \mathbf{Z} \text { for any } i \in J^{\prime} \backslash J \text {. }
$$

By the above calculation, the assertion is further reduced to the case $J^{\prime} \backslash J=\varnothing$ by taking the kernel and cokernel of $\widetilde{\xi}_{i}$ for $i \in J^{\prime} \backslash J$ inductively (and using the $n$-ple complex structure of the Koszul complex). Here we also use the external product $\mathcal{O}_{X_{1}} \times X_{2}=\mathcal{O}_{X_{1}} \otimes \mathcal{O}_{X_{2}}$ together with Theorem 2 if $J^{\prime} \cap J=\varnothing$. We may thus assume that

$$
J^{\prime} \backslash J=\varnothing \text {, that is, } J^{\prime} \subset J .
$$

By a similar argument we may moreover assume that

$$
[1, n] \backslash J=\varnothing, \text { that is, } J=[1, n] .
$$

Note that $n=|J| \geqslant 2$ since $J \backslash J^{\prime} \neq \varnothing$ and $J^{\prime} \neq \varnothing$. 


\section{KONTSEVICH'S CONJECTURE}

Choose $i_{0} \in J \backslash J^{\prime}$. For the calculation of the Koszul complex, we may replace $\widetilde{\xi}_{i}$ with $\widetilde{\xi}_{i}^{\prime}$ defined by

$$
\widetilde{\xi}_{i}^{\prime}:= \begin{cases}\widetilde{\xi}_{i} & \text { if } i=i_{0}, \\ \widetilde{\xi}_{i}-\left(e_{i} / e_{i_{0}}\right) \widetilde{\xi}_{i_{0}} & \text { if } i \neq i_{0}\end{cases}
$$

(since this does not change the vector space spanned by the $\widetilde{\xi}_{i}$ ). For $i \neq i_{0}$, we have

$$
\widetilde{\xi}_{i}^{\prime}=x_{i} \partial_{x_{i}}-\left(e_{i} / e_{i_{0}}\right) x_{i_{0}} \partial_{x_{i_{0}}}-\alpha_{i}^{\prime} \quad \text { with } \alpha_{i}^{\prime} \in \mathbf{C},
$$

and hence

$$
\widetilde{\xi}_{i}^{\prime} x^{\nu}=\left(\nu_{i}-\left(e_{i} / e_{i_{0}}\right) \nu_{i_{0}}-\alpha_{i}^{\prime}\right) x^{\nu}
$$

For $I \subset[1, n] \backslash\left\{i_{0}\right\}$, define

$$
A_{\varepsilon, I}:=\left\{\sum_{\nu \in \mathbf{Z}^{n}} a_{\nu} x^{\nu} \in A_{\varepsilon} \mid a_{\nu}=0 \text { if } \nu_{i} \neq\left(e_{i} / e_{i_{0}}\right) \nu_{i_{0}}+\alpha_{i}^{\prime} \text { for some } i \in I\right\},
$$

and similarly for $A_{\varepsilon, I}^{\prime}$ with $A_{\varepsilon}$ replaced by $A_{\varepsilon}^{\prime}$. Consider a canonical morphism

$$
K^{\bullet}\left(A_{\varepsilon, I}\left(\left(\partial_{t}^{-1}\right)\right), \widetilde{\xi}_{i}\right) \rightarrow K^{\bullet}\left(A_{\varepsilon, I}^{\prime}\left(\left(\partial_{t}^{-1}\right)\right), \widetilde{\xi}_{i}\right) .
$$

By using (2.4.6) together with the $n$-ple complex structure of the Koszul complex, the assertion (2.4.4) is then reduced by increasing induction on $|I|$ to the assertion that the above canonical morphism is a quasi-isomorphism for some $I \subset[1, n] \backslash\left\{i_{0}\right\}$. But when $I=[1, n] \backslash\left\{i_{0}\right\}$, we have the isomorphism

$$
A_{\varepsilon, I} \stackrel{\sim}{\longrightarrow} A_{\varepsilon, I}^{\prime}
$$

Indeed, this easily follows from the condition: $i_{0} \in J \backslash J^{\prime}$. So the assertion is proved for $c=0$, except for the case $J=\varnothing$ (that is, $f=0$ ), which is also needed in our inductive argument. However, the proof in the case $J=\varnothing$ is similar, and is easier since the replacement of $\widetilde{\xi}_{i}$ by $\widetilde{\xi}_{i}^{\prime}$ is not needed in this case. The details are left to the reader.

The assertion for $c=\infty$ is equivalent to the vanishing of the restriction of the left-hand side of the isomorphism in Proposition 4 to $\bar{X}_{\infty}$. In this case, we have $J \subset J^{\prime}$. So the assertion is proved in the same way as in the explanation after (2.4.5) by using the $\partial_{t}^{-1}$-adic filtration. This finishes the proof of Proposition 4.

\section{Proof of the main theorem}

In this section we prove Theorem 1 after showing Propositions 1 and 3 .

\subsection{Proof of Proposition 1}

Using the continuous morphism $\rho: \bar{X}^{\text {an }} \rightarrow \bar{X}$, we get the canonical morphisms

$$
\mathrm{DR}_{\bar{X}}\left(\overline{\mathcal{M}}((u))^{f}\right) \stackrel{\rho^{\#}}{\rightarrow} \rho_{*} \mathrm{DR}_{\bar{X}^{\text {an }}}\left(\overline{\mathcal{M}}^{\text {an }}((u))^{f}\right) \rightarrow \mathbf{R} \rho_{*} \mathrm{DR}_{\bar{X}^{\text {an }}}\left(\overline{\mathcal{M}}^{\text {an }}((u))^{f}\right) .
$$

The middle term is defined by using $\Gamma\left(U^{\text {an }},\left.\Omega_{U^{\text {an }}}^{p} \otimes_{\mathcal{O}_{U} \text { an }} \overline{\mathcal{M}}^{\text {an }}((u))^{f}\right|_{U^{\text {an }}}\right)$ for sufficiently small affine open subvarieties $U$ of $\bar{X}$. Here the noncommutativity of inductive limit and cohomology does not cause any problems for the construction of the morphism $\rho^{\#}$.

Taking the global sections on $\bar{X}$, we get the canonical morphism in Proposition 1. We can then use the truncation $\sigma_{\geqslant p}$, and the assertion is reduced to the case of a sheaf $\mathcal{F}((u))$ with

$$
\mathcal{F}=\overline{\mathcal{M}} \otimes_{\mathcal{O}_{\bar{X}}} \Omega_{\bar{X}}^{p} \quad(p \in \mathbf{Z}) .
$$




\section{Sabbah and M. Saito}

We have the decomposition

$$
\mathcal{F}((u))=\mathcal{F} \llbracket u \rrbracket \oplus \mathcal{F}\left[u^{-1}\right] u^{-1},
$$

and the global cohomology functors on $\bar{X}$ and $\bar{X}^{\text {an }}$ commute with the infinite direct product and also with the infinite direct sum. So the assertion is reduced to the case of $\mathcal{F}$ and follows from GAGA. This finishes the proof of Proposition 1.

\subsection{Proof of Proposition 3}

We may assume that $c=0$. Set

$$
\mathcal{K}_{f}^{\bullet}:=\left.\left(\mathcal{M}\left[\partial_{t}\right]^{f} \otimes_{\mathcal{O}_{X}} \Omega_{X}^{\bullet}\right)\right|_{X_{0}} .
$$

This coincides with the notation in $\S 1.3$ if $\mathcal{M}=\mathcal{O}_{X}$. We have the filtration $V$ on $\mathcal{K}_{f}^{\bullet}$ induced by the filtration $V$ of Kashiwara and Malgrange on $\mathcal{M}\left[\partial_{t}\right]^{f}$, and the assertion is equivalent to the following condition:

$$
\text { The induced filtration } V \text { on } \mathbf{R} \Gamma\left(X_{0}, \mathcal{K}_{f}^{\bullet}\right) \text { is strict. }
$$

We have a spectral sequence of regular holonomic $\mathcal{D}_{S, 0^{-}}$modules

$$
E_{2}^{p, q}=H^{p}\left(X_{0}, \mathcal{H}^{q} \mathcal{K}_{f}^{\bullet}\right) \Rightarrow H^{p+q}\left(X_{0}, \mathcal{K}_{f}^{\bullet}\right) .
$$

This is a filtered spectral sequence since $\mathcal{K}_{f}^{\bullet}$ has the filtration $V$ induced by the filtration $V$ of Kashiwara and Malgrange on $\mathcal{M}\left[\partial_{t}\right]^{f}$. More precisely, let $\mathcal{E}$ denote the category of $\mathbf{C}$-vector spaces having an exhaustive increasing filtration indexed by $\mathbf{Z}$ and let $\mathcal{A}$ be the abelian category consisting of graded vector spaces $\bigoplus_{i \in \mathbf{Z}} E_{i}$ endowed with morphisms $u_{i}: E_{i} \rightarrow E_{i+1}$. Then $\mathcal{E}$ is identified with the full subcategory of $\mathcal{A}$ consisting of subobjects such that the $u_{i}$ are all injective. The spectral sequence (3.2.3) is defined in $\mathcal{A}$, since $V$ is essentially indexed by $m^{-1} \mathbf{Z} \subset \mathbf{Q}$ for some nonzero integer $m$.

By [Del71], 1.3 and [Sai83], 1.3.6, the assertion of Proposition 3 is then reduced to

$$
E_{r}^{p, q} \in \mathcal{E} \quad \text { for any } p, q \in \mathbf{Z} \text { and } r \geqslant 2 \text {. }
$$

We show this by increasing induction on $r \geqslant 2$. Assume that $r=2$. The induced filtration on the cohomology sheaves $\mathcal{H}^{q} \mathcal{K}_{f}^{\bullet}$ is strictly induced, see (1.3.3). It is also strictly induced on $E_{2}^{p, q}=H^{p}\left(X_{0}, \mathcal{H}^{q} \mathcal{K}_{f}^{\bullet}\right)$ by taking the inductive limit for $j \rightarrow-\infty$ of the canonical splitting (1.1.7) which also holds for constructible sheaves of $\mathcal{D}_{S, 0}$-modules. So the assertion is shown for $r=2$.

We can then proceed by increasing induction on $r \geqslant 2$ using the property that the $V$-filtration is strictly compatible with any morphism of regular holonomic $\mathcal{D}_{S, 0}$-modules, see for example [Sai83], 3.1.5. This finishes the proof of Proposition 3.

\subsection{Proof of Theorem 1}

By Propositions 1 and 4, we may replace $X$ and $\mathcal{M}$ with the associated analytic objects. We will denote them by $X$ and $\mathcal{M}$ without adding ${ }^{\text {an }}$. In particular, we consider only analytic sheaves.

We have the filtration $V$ of Kashiwara [Kas83] and Malgrange [Mal83] on $\mathcal{M}\left[\partial_{t}\right]^{f}$ such that the roots of the minimal polynomial of the action of $t \partial_{t}$ on $\operatorname{Gr}_{V}^{i} \mathcal{M}\left[\partial_{t}\right]^{f}$ are contained in $\Lambda+i$, where $\Lambda$ is as in (1.1.4). Set $n=\operatorname{dim} X$. Then we have the canonical isomorphisms

$$
\psi_{f} L[n-1]=\mathrm{DR}_{X}\left(\operatorname{Gr}_{V}^{1} \mathcal{M}\left[\partial_{t}\right]^{f}\right), \quad \varphi_{f} L[n-1]=\mathrm{DR}_{X}\left(\operatorname{Gr}_{V}^{0} \mathcal{M}\left[\partial_{t}\right]^{f}\right)
$$

such that the monodromy $T$ on the left-hand side corresponds to $\exp \left(-2 \pi i t \partial_{t}\right)$ on the right-hand side, see loc. cit. Here $\mathrm{DR}_{X}$ is shifted by $n$ to get perverse sheaves as usual. By Proposition 2 


\section{KontSEviCH's CONJECTURE}

and (1.2.3), the assertion is then reduced to the canonical isomorphisms

$$
\widehat{\mathcal{E}}_{S, 0} \otimes_{\mathcal{D}_{S, 0}} H^{k}\left(X_{0}, \mathcal{K}_{f}^{\bullet}\right) \stackrel{\sim}{\longrightarrow} H^{k}\left(X_{0}, \widehat{\mathcal{E}}_{S, 0} \otimes_{\mathcal{D}_{S, 0}} \mathcal{K}_{f}^{\bullet}\right),
$$

where $\mathcal{K}_{f}^{\bullet}:=\left.\left(\mathcal{M}\left[\partial_{t}\right]^{f} \otimes_{\mathcal{O}_{X}} \Omega_{X}^{\bullet}\right)\right|_{X_{0}}$ as in (3.2.1). Here we may assume that $c=0$ as in $\S 3.1$.

To show (3.3.2), consider the spectral sequence

$$
\widehat{E}_{2}^{p, q}=H^{p}\left(X_{0}, \mathcal{H}^{q}\left(\widehat{\mathcal{E}}_{S, 0} \otimes_{\mathcal{D}_{S, 0}} \mathcal{K}_{f}^{\bullet}\right)\right) \Rightarrow H^{p+q}\left(X_{0}, \widehat{\mathcal{E}}_{S, 0} \otimes_{\mathcal{D}_{S, 0}} \mathcal{K}_{f}^{\bullet}\right)
$$

We have a canonical morphism from the spectral sequence (3.2.3) to (3.3.3). So it is enough to show that it induces the isomorphisms

$$
\widehat{\mathcal{E}}_{S, 0} \otimes_{\mathcal{D}_{S, 0}} E_{r}^{p, q} \stackrel{\sim}{\longrightarrow} \widehat{E}_{r}^{p, q} .
$$

For $r=2$, the isomorphism follows from (1.2.3). We can then proceed by increasing induction on $r \geqslant 2$ since $\widehat{\mathcal{E}}_{S, 0}$ is flat over $\mathcal{D}_{S, 0}$. So (3.3.4) and then (3.3.3) follow. This finishes the proof of Theorem 1.

\section{4 Čech calculation}

Let $\left\{U_{i}\right\}$ be an affine open covering of $X$. Set $U_{I}:=\bigcap_{i \in I} U_{i}$ for $I \neq \varnothing$. Then the left-hand side of the formula in Theorem 1 is given by the $k$-th cohomology of the single complex associated with a double complex whose $(p, q)$-component is

$$
\bigoplus_{|I|=p+1} \Gamma\left(U_{I},\left.\mathcal{M} \otimes \mathcal{O}_{X} \Omega_{X}^{q}\right|_{U_{I}}\right)((u))^{f},
$$

where the first and second differentials are given by the Čech differential and $\nabla-u^{-1} d f \wedge$, respectively.

\subsection{Isolated singularity case}

If $f$ has only isolated singular points, then the filtration $F$ is strict, that is, we have the $E_{1}$ degeneration of the spectral sequence associated with the filtration $F$ on the left-hand side of the formulas in Theorems 1 and 2, where $F$ is defined by a natural generalization of (1.5.2) to the local system case. In fact, their $E_{0}$-complexes are essentially given by the Koszul complexes associated with $d f \wedge$, which are acyclic except for the top degree. This implies that we get the $\partial_{t}^{-1}$-adic completion of the Gauss-Manin system at each singular point of $f$. The filtration $F$ on it is given by applying $\partial_{t}^{k}(k \in \mathbf{Z})$ to the completion of the Brieskorn lattice up to a shift by $\operatorname{dim} X$. See also Remark 1.7(i) for the relation with [Sai88a].

\subsection{Remark}

Let $V_{c}^{k}, V^{k}, A_{1}(c, c)$ be as in the remark after Theorem 1. By $\left[t, \partial_{t}^{-i}\right]=i \partial_{t}^{-i-1}$, we can modify $V^{k}$ with $\widehat{R} V^{k}$ and $A_{1}(c, c)$ unchanged, so that by increasing induction on $i \geqslant 1$, we have

$$
(t-c) V_{c}^{k} \subset \partial_{t}^{-1} \widehat{R} V_{c}^{k}+\partial_{t}^{-i-1} \widehat{R} V^{k} .
$$

In fact, if $\left\{v_{c, j}\right\}$ is a basis of $V_{c}^{k}$, then we can replace $v_{c, j}$ with

$$
v_{c, j}+\sum_{j^{\prime}, c^{\prime} \neq c} a_{c, j, c^{\prime}, j^{\prime}} \partial_{t}^{-i} v_{c^{\prime}, j^{\prime}}
$$

by increasing induction on $i \geqslant 1$, where the $a_{c, j, c^{\prime}, j^{\prime}} \in \mathbf{C}$ are chosen appropriately. Passing to the limit, this means that $A_{i}\left(c, c^{\prime}\right)=0$ for $c \neq c^{\prime}$. The conclusion of the remark after Theorem 1 then follows. 


\section{Sabbah And M. Saito}

\section{Generalization to the regular holonomic case}

In this section we show how to generalize the arguments in the previous sections in order to prove Theorem 3.

\subsection{Gauss-Manin systems with coefficients}

Let $X$ be a complex manifold and let $E$ be a divisor with simple normal crossings on it. Let $\mathcal{M}$ be a locally free $\mathcal{O}_{X}$-module of finite type endowed with an integrable regular singular connection $\nabla$ having simple poles along $E$ such that the real part of any eigenvalue of the residue of $\nabla$ along each irreducible component of $E$ is contained in $[0,1)$. Let $\mathcal{M}(* E)$ be the localization of $\mathcal{M}$ along $E$. This is a regular holonomic left $\mathcal{D}_{X}$-module. Let $L$ be the local system on $X \backslash E$ corresponding to $\left.\mathcal{M}\right|_{X \backslash E}$. We have the canonical isomorphisms in $D_{c}^{b}(X, \mathbf{C})$ :

$$
\mathbf{R} j_{*}^{\prime} L \stackrel{\sim}{\longrightarrow} \mathcal{M} \otimes_{\mathcal{O}_{X}} \Omega_{X}^{\bullet}(\log E) \stackrel{\sim}{\longrightarrow} \mathcal{M}(* E) \otimes_{\mathcal{O}_{X}} \Omega_{X}^{\bullet},
$$

where $j^{\prime}: X \backslash E \hookrightarrow X$ is the inclusion, see [Del70].

Let $f$ be a holomorphic function on $X$ with $X_{0}:=f^{-1}(0) \subset E$. The direct image as a left $\mathcal{D}$-module of $\mathcal{M}(* E)$ by the graph embedding of $f$ is identified with

$$
\mathcal{M}(* E)\left[\partial_{t}\right]^{f},
$$

where ${ }^{f}$ means that the action of $\mathcal{D}_{X}$ and $t$ are twisted as in $\S 1$.3. Set

$$
\mathcal{K}_{\mathcal{M}, f}^{\bullet}:=\left.\left.\mathcal{M}\left[\partial_{t}\right]^{f} \otimes_{\mathcal{O}_{X}} \Omega_{X}^{\bullet}(\log E)\right|_{X_{0}} \subset \mathcal{M}(* E)\left[\partial_{t}\right]^{f} \otimes_{\mathcal{O}_{X}} \Omega_{X}^{\bullet}\right|_{X_{0}}
$$

Here the last complex is the relative de Rham complex associated with $\mathcal{M}(* E)\left[\partial_{t}\right]^{f}$, and the last inclusion is a quasi-isomorphism. The Gauss-Manin systems with coefficients in $\mathcal{M}$ are defined by

$$
\mathcal{G}_{\mathcal{M}, f}^{i}:=\mathcal{H}^{i} \mathcal{K}_{\mathcal{M}, f}
$$

These are constructible sheaves of regular holonomic $\mathcal{D}_{S, 0}$-modules on $X_{0}$ as in $\S 1.3$, and

$$
\left(\mathcal{G}_{\mathcal{M}, f}^{i+1}\right)^{\alpha}=\mathcal{H}^{i} \psi_{f, \lambda} \mathbf{R} j_{*}^{\prime} L \text { with } \lambda:=\exp (-2 \pi i \alpha),
$$

where the left-hand side is defined by $\operatorname{Ker}\left(t \partial_{t}-\alpha\right)^{k} \subset \mathcal{G}_{\mathcal{M}, f}^{i+1}$ for $k \gg 0$ as in $\S 1.1$. Note that in this case, $\psi_{f, \lambda} \mathbf{R} j_{*}^{\prime} L=\varphi_{f, \lambda} \mathbf{R} j_{*}^{\prime} L$. Moreover, the filtration $V$ on the $\mathcal{G}_{\mathcal{M}, f}^{i}$ is induced by the filtration $V$ on the $\mathcal{D}$-module $\mathcal{M}(* E)\left[\partial_{t}\right]^{f}$. These can be shown by using the Milnor fibration around each point of $X_{0}$, see for example [Sai88b], Prop. 3.4.8.

Define a subcomplex $\mathcal{A}_{\mathcal{M}, f}^{\bullet} \subset \mathcal{K}_{\mathcal{M}, f}^{\bullet}$ by

$$
\mathcal{A}_{\mathcal{M}, f}^{i}:=\left.\operatorname{Ker}\left(d f \wedge: \mathcal{M} \otimes \mathcal{O}_{X} \Omega_{X}^{i}(\log E) \rightarrow \mathcal{M} \otimes_{\mathcal{O}_{X}} \Omega_{X}^{i+1}(\log E)\right)\right|_{X_{0}} .
$$

We show that $\mathcal{H}^{i}\left(\mathcal{A}_{\mathcal{M}, f}^{\bullet}\right)_{x}$ for $x \in X_{0}$ is a finite free $\mathbf{C}\{t\}$-submodule of $\left(\mathcal{G}_{\mathcal{M}, f}^{i}\right)_{x}$ which generates it over $\mathcal{D}_{S, 0}$ and is stable by the action of $t \partial_{t}$, so that the real part of any eigenvalue of the residue of $t \partial_{t}$ is contained in $[-1,0)$. Setting $\lambda:=\exp (-2 \pi i \alpha)$, by (4.1.1) this assertion is equivalent to the following:

$$
\left(\mathcal{H}^{i+1} \mathcal{A}_{\mathcal{M}, f}\right)^{\alpha}= \begin{cases}\mathcal{H}^{i} \psi_{f, \lambda} \mathbf{R} j_{*}^{\prime} L & \text { if } \alpha \geqslant 0, \\ 0 & \text { if } \alpha<-1 .\end{cases}
$$

Using this, we can define the filtration $G$ on $\mathcal{K}_{\mathcal{M}, f}^{\bullet}$ and $\mathcal{K}_{\mathcal{M}, f}\left[\partial_{t}^{-1}\right]$ in the same way as (1.5.2).

To show assertion (4.1.2), consider the relative logarithmic de Rham complex

$$
\mathcal{A}_{\mathcal{M}, f}^{\prime}:=\mathcal{M} \otimes \mathcal{O}_{X} \Omega_{X / S}^{\bullet}(\log E),
$$




\section{KontSEviCH's CONJECTURE}

where

$$
\Omega_{X / S}^{i}(\log E):=\Omega_{X}^{i}(\log E) /\left(d f / f \wedge \Omega_{X}^{i-1}(\log E)\right) .
$$

There is an isomorphism of complexes

$$
d f / f \wedge: \mathcal{A}_{\mathcal{M}, f}^{\prime \bullet} \stackrel{\sim}{\longrightarrow} \mathcal{A}_{\mathcal{M}, f}[1]
$$

by using the acyclicity of the complex $\left(\Omega_{X}^{\bullet}(\log E), d f / f \wedge\right)$ together with the relation

$$
d(d f / f \wedge \omega)=-d f / f \wedge d \omega \text { for } \omega \in \Omega_{X}^{i}(\log E) .
$$

The following is known to the specialists:

(4.1.4) The action of $t \partial_{t}$ on $\mathcal{H}^{i} \mathcal{A}_{\mathcal{\mathcal { M }}, f}^{\prime}$ corresponds to that of $\partial_{t} t=t \partial_{t}+1$ on $\mathcal{H}^{i+1} \mathcal{A}_{\mathcal{M}, f}$ under the isomorphism induced by (4.1.3).

Indeed, the action of $t \partial_{t}$ on $\mathcal{H}^{i} \mathcal{A}_{\mathcal{M}, f}^{\prime}$ and that of $\partial_{t} t$ on $\mathcal{H}^{i+1} \mathcal{A}_{\mathcal{M}, f}$ can be defined by using the boundary morphism of the long exact sequence associated with the short exact sequence of complexes

$$
0 \rightarrow \mathcal{A}_{\mathcal{M}, f}^{\bullet} \rightarrow \mathcal{M} \otimes_{\mathcal{O}_{X}} \Omega_{X}^{\bullet}(\log E) \rightarrow \mathcal{A}_{\mathcal{M}, f}^{\prime} \rightarrow 0
$$

together with the isomorphism (4.1.3). More explicitly, for $\left[\omega^{\prime}\right] \in \mathcal{H}^{i} \mathcal{A}_{\mathcal{M}, f}^{\prime}$, we have

$$
t \partial_{t}\left[\omega^{\prime}\right]=\left[\eta^{\prime}\right] \text { if }(d f / f) \wedge \eta^{\prime}=\nabla \omega^{\prime},
$$

and for $[\omega] \in \mathcal{H}^{i+1} \mathcal{A}_{\mathcal{M}, f}$, we have

$$
\partial_{t} t[\omega]=[\nabla \eta] \text { if }(d f / f) \wedge \eta=\omega
$$

where $[\omega]$ denotes the class of $\omega$, etc. So we get (1.4.4). These actions are compatible with the $\mathcal{D}_{S, 0^{-}}$ module structure on the Gauss-Manin systems $\mathcal{G}_{\mathcal{M}, f}^{i}$ by the canonical morphism $\mathcal{A}_{\mathcal{M}, f}^{\bullet} \hookrightarrow \mathcal{K}_{\mathcal{M}, f}$. Note that $d f / f \wedge($ instead of $d f \wedge)$ is used in the above argument. This gives the difference between $t \partial_{t}$ and $\partial_{t} t$, and also $[0,1)$ and $[-1,0)$ before (4.1.2).

The proof of (4.1.2) for $\mathcal{H}^{i} \mathcal{A}_{\mathcal{M}, f}^{\prime}$ (with -1 replaced by 0 ) can be reduced to the case $\operatorname{rank} \mathcal{M}=1$ by increasing induction on $\operatorname{rank} \mathcal{M}$ using a long exact sequence associated with a short exact sequence of locally free sheaves with regular singular integrable connection, since the assertion is local. If $\operatorname{rank} \mathcal{M}=1$, we can calculate $\mathcal{H}^{i} \mathcal{A}_{\mathcal{M}, f}^{\prime}$ explicitly by using a Koszul complex as in [Ste76], see $\S 4.2$ below.

\subsection{Rank 1 case}

With the above notation and assumption, assume that $\operatorname{rank} \mathcal{M}=1$. Let $x_{1}, \ldots, x_{n}$ be local coordinates of $X$ such that locally, we have

$$
f=\prod_{i \in J} x_{i}^{e_{i}}, \quad E=\bigcup_{i \in J^{\prime}}\left\{x_{i}=0\right\},
$$

where $J \subset J^{\prime}$ and $e_{i} \geqslant 1$ for $i \in J$. Then the logarithmic de Rham complex $\mathcal{M} \otimes_{\mathcal{O}_{X}} \Omega_{X}^{\bullet}(\log E)$ is locally the Koszul complex associated with the operators $\xi_{i}$ on $\mathcal{O}_{X}$ for $i \in[1, n]$, defined by

$$
\xi_{i}= \begin{cases}x_{i} \partial_{x_{i}}+\alpha_{i} & \text { if } i \in J^{\prime}, \\ \partial_{x_{i}} & \text { if } i \notin J^{\prime},\end{cases}
$$

where $\alpha_{i} \in \mathbf{C}$ and its real part is contained in [0,1). The relative logarithmic de Rham complex $\mathcal{A}_{\mathcal{M}, f}^{\prime}=\mathcal{M} \otimes_{\mathcal{O}_{X}} \Omega_{X / S}^{\bullet}(\log E)$ is the quotient complex defined by the relation

$$
\sum_{i=1}^{r^{\prime}} e_{i}\left[d x_{i} / x_{i}\right]=0,
$$




\section{Sabbah and M. Saito}

where $\left[d x_{i} / x_{i}\right]$ denotes the class of $d x_{i} / x_{i}$. Choose $i_{0} \in J$. Then $\mathcal{A}_{\mathcal{M}, f}^{\prime}$ is locally the Koszul complex associated with the operators $\widetilde{\xi}_{i}$ on $\mathcal{O}_{X}$ for $i \in[1, n] \backslash\left\{i_{0}\right\}$, defined by

$$
\widetilde{\xi}_{i}= \begin{cases}\left(x_{i} \partial_{x_{i}}+\alpha_{i}\right)-\left(e_{i} / e_{i_{0}}\right)\left(x_{i_{0}} \partial_{x_{i_{0}}}+\alpha_{i_{0}}\right) & \text { if } i \in J \backslash\left\{i_{0}\right\}, \\ x_{i} \partial_{x_{i}}+\alpha_{i} & \text { if } i \in J^{\prime} \backslash J, \\ \partial_{x_{i}} & \text { if } i \notin J^{\prime}\end{cases}
$$

This implies that $\mathcal{H}^{p}\left(\mathcal{A}_{\mathcal{M}, f}^{\prime}\right)_{0}=0$ when $\alpha_{i} \neq 0$ for some $i \in J^{\prime} \backslash J$, since $\operatorname{Re} \alpha_{i} \in[0,1)$. We may thus assume that

$$
\alpha_{i}=0 \text { for any } i \in J^{\prime} \backslash J \text {. }
$$

Set

$$
A_{0}:=\left\{\sum_{\nu \in \mathbf{N}^{J}} a_{\nu} x^{\nu} \in \mathbf{C}\left\{x_{J}\right\} \mid \nu_{i}+\alpha_{i}=\left(e_{i} / e_{i_{0}}\right)\left(\nu_{i_{0}}+\alpha_{1}\right)\left(\forall i \in J \backslash\left\{i_{0}\right\}\right)\right\},
$$

where $\mathbf{C}\left\{x_{J}\right\}:=\mathbf{C}\left\{x_{i}(i \in J)\right\}$ and $\nu=\left(\nu_{i}\right)_{i \in J} \in \mathbf{N}^{J}$. We then get

$$
\mathcal{H}^{p}\left(\mathcal{A}_{\mathcal{M}, f}^{\prime}\right)_{0}=\bigoplus A_{0}\left[d x_{i_{1}} / x_{i_{1}}\right] \wedge \cdots \wedge\left[d x_{i_{p}} / x_{i_{p}}\right]
$$

where the direct sum is taken over $\left\{i_{1}, \ldots, i_{p}\right\} \subset J \backslash\left\{i_{0}\right\}$ with $i_{1}<\cdots<i_{p}$. This is a generalization of [Ste76] which treated the constant local system case.

We can calculate the action of $t \partial_{t}$ on

$$
\mathbf{C} x^{\nu}\left[d x_{i_{1}} / x_{i_{1}}\right] \wedge \cdots \wedge\left[d x_{i_{p}} / x_{i_{p}}\right] \subset \mathcal{H}^{p}\left(\mathcal{A}_{\mathcal{M}, f}^{\prime}\right)_{0}
$$

with $x^{\nu} \in A_{0}$. By the argument in $\S 4$.1, this is given by the multiplication by

$$
\left(\nu_{i}+\alpha_{i}\right) / e_{i},
$$

which is independent of $i \in J$ by the definition of $A_{0}$.

The localization of $\mathcal{H}^{p}\left(\mathcal{A}_{\mathcal{M}, f}^{\prime}\right)_{0}$ by $t$ is the localization of $\mathcal{A}_{\mathcal{M}, f}^{\prime}$ by $f$, and the above calculation can be extended to the localization. Here the real part of $\left(\nu_{i}+\alpha_{i}\right) / e_{i}$ is strictly negative when $\nu_{i} \in \mathbf{Z}_{<0}$, since $\operatorname{Re} \alpha_{i} \in[0,1)$. This implies that $\mathcal{H}^{p}\left(\mathcal{A}_{\mathcal{M}, f}^{\prime}\right)_{0}$ is a finite free $\mathbf{C}\{t\}$-module which is stable by the action of $t \partial_{t}$ and such that the real part of any eigenvalue of the residue of $t \partial_{t}$ is contained in $[0,1)$. This finishes the proof of (4.1.2).

\subsection{Proof of Theorem 3}

The canonical morphisms of Theorems 2 and 3 are constructed in the same way as the local system case. The assertions are then reduced to the case where $\mathcal{M}$ is a regular holonomic $\mathcal{D}$ module. Let $E$ be a divisor on $X$ such that the restriction of $\operatorname{DR}(\mathcal{M})$ to the complement of $E$ is a local system supported on a smooth variety. Let $i: E \hookrightarrow X$ and $j: X \backslash E \hookrightarrow X$ denote the inclusions. We have a distinguished triangle

$$
i_{*} i^{!} \mathcal{M} \rightarrow \mathcal{M} \rightarrow \mathbf{R} j_{*} j^{*} \mathcal{M} \rightarrow
$$

By increasing induction on $\operatorname{dim} \operatorname{supp} \mathcal{M}$, the assertions are then reduced to the case where $\mathcal{M}=$ $\mathbf{R} j_{*} j^{*} \mathcal{M}$ (using the canonical morphisms mentioned above). Here we may assume that $\mathcal{M}$ is supported on $X$ and $D \cup E$ is a divisor with simple normal crossings on $\bar{X}$ by replacing $\bar{X}$ with a desingularization of the closure of the support of $\mathcal{M}$ in $\bar{X}$, if necessary (using the compatibility with the direct image explained at the end of $\S 2.1$ ). Note that the assertions are easy to show when the support of $\mathcal{M}$ is contained in a fiber of $f$. Replacing $E$ with a larger divisor and 


\section{Kontsevich's CONJECTURE}

blowing $\bar{X}$ up further if necessary, we may moreover assume that

$$
\bar{f}(\operatorname{Sing} \bar{f}) \cap f(E)=\varnothing ;
$$

if $E$ contains a divisor $Z$ of $X$ with $f(Z)$ a point, then $E$ contains $f^{-1} f(Z)$.

Then Theorem 3 follows since the proofs of Propositions 2, 3, and 4 in the local system case are generalized to this situation by using the arguments in $\S 4.1-4.2$. For instance, in the generalization of $\S 2.4$ with $\operatorname{rank} \mathcal{M}=1$, there are three subsets $J, J^{\prime}, J^{\prime \prime}$ of $[1, n]$ corresponding to $f, D$, and the closure of $E$, respectively, where $J^{\prime} \cap J^{\prime \prime}=\varnothing$ and $J \subset J^{\prime} \cup J^{\prime \prime}$ by (4.3.2). If $J \neq \varnothing$ (that is, if $f \neq 0$ ), then we can reduce to the case $J=J^{\prime} \cup J^{\prime \prime}=[1, n]$ with $J^{\prime}$ nonempty by the same argument as in $\S 2.4$. The argument for the case $J=\varnothing$ is similar and easier. The details are left to the reader. This finishes the proof of Theorem 3.

\section{REFERENCES}

BS07 D. Barlet and M. Saito, Brieskorn modules and Gauss-Manin systems for non-isolated hypersurface singularities, J. London Math. Soc. (2) 76 (2007), 211-224. http://dx.doi.org/10.1112/ jlms/jdm027

Bri70 E. Brieskorn, Die Monodromie der isolierten Singularitäten von Hyperflächen, Manuscripta Math. 2 (1970), 103-161.

Del70 P. Deligne, Equations différentielles à points singuliers réguliers, Lect. Notes in Math. 163, Springer, Berlin (1970).

Del71 P. Deligne, Théorie de Hodge, II, Inst. Hautes Études Sci. Publ. Math. 40 (1971), 5-57.

Del73 P. Deligne, Le formalisme des cycles évanescents, in SGA7 XIII and XIV, Lect. Notes in Math. 340, Springer, Berlin (1973), 82-115 and 116-164.

GM80 M. Goresky and R. MacPherson, Intersection homology theory, Topology 19 (1980), 135-162.

Kas83 M. Kashiwara, Vanishing cycle sheaves and holonomic systems of differential equations, Lect. Notes in Math. 1016, Springer, Berlin (1983), 134-142.

Kas03 M. Kashiwara, D-modules and microlocal calculus, Translations of Mathematical Monographs 217, American Mathematical Society, Providence, RI (2003).

KS11 M. Kontsevich and Y. Soibelman, Cohomological Hall algebra, exponential Hodge structures and motivic Donaldson-Thomas invariants, Commun. Number Theory Phys. 5 (2011), 231-352. http: //dx.doi.org/10.4310/CNTP.2011.v5.n2.a1

Mal83 B. Malgrange, Polynôme de Bernstein-Sato et cohomologie évanescente, Analysis and topology on singular spaces, II, III (Luminy, 1981), Astérisque 101-102 (1983), 243-267.

Sab10 C. Sabbah, On a twisted de Rham complex, II, arXiv:1012.3818 (2010).

Sai83 M. Saito, Hodge filtrations on Gauss-Manin systems, II, Proc. Japan Acad. Ser. A Math. Sci. 59 (1983), 37-40.

Sai88a M. Saito, Exponents and Newton polyhedra of isolated hypersurface singularities, Math. Ann. 281 (1988), 411-417.

Sai88b M. Saito, Modules de Hodge polarisables, Publ. RIMS, Kyoto Univ. 24 (1988), 849-995.

Sai89 M. Saito, Induced D-modules and differential complexes, Bull. Soc. Math. France 117 (1989), 361-387.

SKK73 M. Sato, T. Kawai, and M. Kashiwara, Microfunctions and pseudo-differential equations, Lect. Notes in Math. 287, Springer, Berlin (1973), 265-529.

Ste76 J.H.M. Steenbrink, Limits of Hodge structures, Inv. Math. 31 (1976), 229-257. 


\section{Sabbah and M. Saito}

Claude Sabbah

UMR 7640 du CNRS, Centre de Mathématiques Laurent Schwartz, École polytechnique, 91128

Palaiseau cedex, France

Morihiko Saito

RIMS Kyoto University, Kyoto 606-8502, Japan 